\title{
Blackberry phenolic and volatile extracts inhibit cytokine secretion in LPS-inflamed RAW264.7 cells
}

\author{
Pauline Morin ${ }^{\mathrm{a}}$, Luke Howard ${ }^{\mathrm{a}}$, John Tipton ${ }^{\mathrm{b}}$, Laura Lavefve ${ }^{\mathrm{a}}$, Cindi Brownmiller ${ }^{\mathrm{a}}$, \\ Sun-Ok Lee ${ }^{\mathrm{a}}$, Inah $\mathrm{Gu}^{\mathrm{a}}$, Rohana Liyanage ${ }^{\mathrm{c}}$ and Jackson O. Lay ${ }^{\mathrm{c}}$
}

\begin{abstract}
aDepartment of Food Science, University of Arkansas, 2650 N. Young Ave., Fayetteville, AR 72704, USA
${ }^{b}$ Mathematical Sciences, Science Engineering Hall, 850 West Dickson Street, Fayetteville, AR 72701, USA

'University of Arkansas Statewide Mass Spectrometry Facility, 1260 West Maple Street, Fayetteville, AR 72701, USA

*Corresponding author: Luke R. Howard, Department of Food Science, University of Arkansas, 2650 N. Young Ave., Fayetteville, AR 72704, USA. Tel: (479) 575-2968; Fax: (479) 575-6936; E-mail: lukeh@uark.edu
\end{abstract}

DOI: $10.31665 /$ JFB.2021.16291

Received: December 06, 2021; Revised received \& accepted: December 30, 2021

Abbreviations: C3GE, Cyanidin 3-glucoside equivalents; DPPH, 2,2 diphenyl-1-picrylhydrazyl; ELISA, Enzyme linked immunosorbent assay; GAE, Gallic acid equivalents; GC, Gas chromatography; GC-FID, Gas chromatography - flame ionization detection; GC-MS, Gas chromatography - mass spectrometery; HPLC, High pressure liquid chromatography; HPLC-MS, High pressure liquid chromatography mass spectrometry; IL-6, Interluekin 6; LPS, Lipopolysaccharide; MAPK, Mitogen-activated protein kinase; NC, Negative control; NED, $\mathrm{N}-1$-napthylethylenediamine dihydrochloride; NF- $\mathrm{B}$, Nuclear factor of kappa light polypeptide gene enhancer in beta cells; NO, Nitric oxide; Phe, Phenolic extract; RE, Rutin equivalents; SPE, Solid phase extraction; SPME, Solid phase microextraction; TNF $\alpha$, Tumor necrosis factor alpha; Vol, Volatile extract

Citation: Morin, P., Howard, L., Tipton, J., Lavefve, L., Brownmiller, C., Lee, S.-O., Gu, I., Liyanage, R., and Lay, J.O. (2021). Blackberry phenolic and volatile extracts inhibit cytokine secretion in LPS-inflamed RAW264.7 cells. J. Food Bioact. 16: 34-47.

\begin{abstract}
The anti-inflammatory activity of blackberries has been attributed to phenolic compounds, especially anthocyanins. The present study hypothesized that volatiles could contribute to anti-inflammatory activity as well. The anti-inflammatory properties of three blackberry genotypes varying in total volatile and phenolic contents were assessed by measuring concentrations of nitric oxide (NO), interleukin- 6 (IL-6) and tumor necrosis factor- $\alpha$ (TNF- $\alpha$ ) within LPS-inflamed RAW264.7 murine macrophage cells after a preventive treatment of either a phenolic or a volatile extract. Extracts from blackberry genotypes A2528T, A2587T and Natchez had total phenolic contents of $4,315,3,369$ and $3,680 \mu \mathrm{g} / \mathrm{mL}$, respectively, and total volatile contents of 283,852 and $444 \mathrm{ng} / \mathrm{mL}$, respectively. Phenolic and volatile extracts of all genotypes significantly lowered the secretion of NO, IL- 6 and TNF- $\alpha$ in ranges varying between $20-42 \%, 34-60 \%$ and $28-73 \%$ inhibition, respectively. Volatile extracts exhibited greater anti-inflammatory properties than phenolic extracts, despite being present at much lower concentrations in the berries. Further research is needed to assess bioavailability and anti-inflammatory effect of blackberry volatiles in vivo.
\end{abstract}

Keywords: Anti-inflammatory; Blackberries; Cytokines; Inflammation; In vitro; Phenolics.

\section{Introduction}

With $67 \%$ of their worldwide acreage based in the United States, blackberries (Rubus subgenus Rubus Watson) are becoming more common in the American diet (Strick, 2007). Blackberries are known for many health-promoting properties such as antioxidant, anticancer and anti-inflammatory capacities (Seeram et al., 2006; Bowen-Forbes et al., 2010; Van de Velde et al., 2016; Van de Velde et al., 2019). Blackberries contain a variety of phenolic compounds, anthocyanins, flavonols, ellagitannins, and phenolic acids responsible for bioactive properties and health benefits (Kaume et al., 2012). Anthocyanins in particular, which are responsible for 
berry colors, are often the focus of the studies since they are the major blackberry phytochemical (Strick, 2007).

Blackberries contain a wide array of volatile compounds including acids, alcohols, esters, monoterpenes and sesquiterpenes that contribute to the berries aroma (Du et al., 2010; Qian and Wang, 2005). Little research has been conducted on the anti-inflammatory effect of blackberry volatiles, but studies have demonstrated the anti-inflammatory capacities of essential oils that contain common blackberry volatiles (Hirota et al., 2010; Huo et al., 2013; Plastina et al., 2018). Moreover, in a recent study by Moore et al, (2019), phenolic and volatile extracts isolated from cranberries, as well as each of the four most abundant volatiles in cranberries, $\alpha$-terpeniol, linalool oxide, eucalyptol, and linalool were tested at concentrations found in fresh cranberries for their anti-inflammatory capacities, before or after inflammation of RAW264.7 murine macrophage cells. When applied as a treatment after induction of inflammation in the cells, not only did the cranberry volatiles decrease nitric oxide (NO) production by the inflamed cells, but their effect was comparable to that of cranberry phenolics, even though they were 353 times less concentrated. When used as a preventive treatment, before induction of inflammation in the cells, both phenolic and volatile extracts showed stronger NO reduction in comparison with treatment after inflammation, exerting comparable NO production between the two extracts. In another recent study by $\mathrm{Gu}$ et al. (2020), phenolic and volatile fractions from blackberries, blueberries, red raspberries, strawberries, cranberries and black raspberries showed comparable ability to inhibit lipopolysaccharide (LPS)-induced production of NO, prostaglandin $\mathrm{E}_{2}$, cyclooxygenase-2, interleukin-6 (IL-6) and tumor necrosis factor- $\alpha$ $(\mathrm{TNF}-\alpha)$. Additionally, the berry phenolic and volatile extracts reduced nuclear translocation of nuclear factor kappa B (NF- $\kappa \mathrm{B})$ by blocking the degradation of $\mathrm{I} \kappa \mathrm{B} \alpha$ and subsequent phosphorylation of $\mathrm{p} 65$. Based on these findings, our hypothesies were 1) blackberry volatile compounds contribute to the anti-inflammatory capacities of blackberries, and 2) blackberry genotypes varying in volatile composition will possess varying anti-inflammatory capacities. To investigate these hypotheses we evaluated three blackberry genotypes varying in phenolic and volatile composition for their ability to inhibit the secretion of NO, IL- 6 and TNF- $\alpha$ after inflammation of RAW264.7 murine macrophage cells.

\section{Materials and methods}

\subsection{Chemicals and reagents}

2-heptanol, ethyl acetate, $\alpha$-terpineol, terpinen-4-ol, $\alpha$-p-dimethylstyrene, 1-octanol, 1-hexanol, geraniol, $\beta$-myrcene, 3-hexan-1-ol, perillic alcohol, myrtenol, linalool, thymol, D-limonene, decanal, 2-hexenal, nerol, nonanal, $\alpha$-terpinene, 1-pentanol, borneol, hexanal, citronellol, pentanal, benzaldehyde, 1-butanol-3-methyl, octanal, ethyl hexanoate, toluene, 2-decaenal, 1-nonanol, cymene, 1-heptanol, 2-heptanone, 1-octen-3-ol, 3-hexen-1-ol, 1-decanol, 5-hepten-2-one, methyl, methyl octanoate, $\beta$-ionone, 2-octanal, heptanal, Folin-Ciocalteu reagent, gallic acid, rutin, alkane standards (C5-C20), HPLC grade methanol, acetone, acetic acid, formic acid, Tween-20, trolox, 2,2 diphenyl-1-picrylhydrazyl (DPPH) and lipopolysaccharide (E. Coli 0111 :B4) were obtained from SigmaAldrich (St. Louis, MO). Cyanidin 3-glucoside was obtained from Polyphenols AS (Sandnes, Norway). Sep-Pak ${ }^{\circledR} \mathrm{C}_{18}$ cartridges were obtained from Waters Corp. (Milford, MA). Dulbecco's modified Eagle's medium (DMEM), fetal bovine serum (FBS), phosphate buffered saline (PBS, pH 7.2), and other cell culture reagents were purchased from Thermo Fischer Scientific (Waltham, MA). 3-(4,5-dimethylthiazol-2-yl)-5-(3 carboxymethoxyphenyl)2-(4-sulfophenyl)-2H-tetrazolium (MTS) and Griess reagent system were obtained from Promega Co. (Madison, WI). Enzyme-linked immunosorbent assays (ELISA) kits used to measure levels of IL-6 and TNF- $\alpha$ (mouse IL- 6 ELISA and mouse TNF- $\alpha$ ELISA) were obtained from RayBiotech Inc. (Norcross, GA).

\subsection{Plant material}

One named-variety (Natchez) and two advanced University of Arkansas breeding lines (A2528T, A2587T) were harvested at the University of Arkansas Fruit Research Station in Clarksville, AR. Fruits were picked when they were fully black and based on ease of abscission from pedicel, according to the recommendation of Perkins-Veazie et al. (1996). The berries were transported in coolers to the Department of Food Science, and upon arrival were placed into sealable Ziploc bags and stored at $-20{ }^{\circ} \mathrm{C}$ until further analysis.

\subsection{Chemical characterization}

\subsubsection{Extraction and purification of blackberry phenolics}

Due to varying berry sizes among genotypes, blackberries were sliced longitudinally and a sample of $50 \mathrm{~g}$ of half-fruits was weighted. Then, blackberries were homogenized with an IKA T-18 UltraTurrax mixer (Wilmington, NC) in $100 \mathrm{~mL}$ of extraction solvent containing methanol, water and formic acid $(60: 37: 3 \mathrm{v} / \mathrm{v} / \mathrm{v})$ and then centrifuged at $6,000 \mathrm{~g}$ for $5 \mathrm{~min}$. The supernatant was filtered through MiraCloth (CalBiochem, LaJolla, CA) into a flask, and the pellet was re-extracted with $100 \mathrm{~mL}$ of acetone, water and acetic acid $(70: 29.5: 0.5 \mathrm{v} / \mathrm{v} / \mathrm{v})$ before being centrifuged at 6,000 $\mathrm{g}$ for 5 $\mathrm{min}$. These steps were repeated until the sediments recovered from the MiraCloth and the pellet following centrifugation had no visible color. The sediments and MiraCloth were then rinsed with the solvents into the flask containing all supernatants. Samples were then adjusted with extraction solvent to $500 \mathrm{~mL}$ in a volumetric flask. The extraction was repeated three times for each genotype.

The phenolic extracts were subjected to Solid Phase Extraction (SPE) in order to remove non-phenolic compounds prior to use for cell culture. A volume of $50 \mathrm{~mL}$ of phenolic extract, which equates to $5 \mathrm{~g}$ of fresh berries, was dried using a Speed-Vac ${ }^{\circledR}$ Plus SC210A concentrator overnight. The samples were then solubilized in $5 \mathrm{~mL}$ of DI water. The SPE unit was equipped with Sep-Pak ${ }^{\circledR} \mathrm{C}_{18}$ cartridges (Waters, Milford, MA) for each sample. Each cartridge was preconditioned with $20 \mathrm{~mL}$ of $80 \%$ methanol and $40 \mathrm{~mL}$ of DI water before the sample $(5 \mathrm{~mL})$ was loaded. The cartridge was then rinsed with $40 \mathrm{~mL}$ DI water and the eluate was discarded. Next, the cartridge was rinsed with $80 \mathrm{~mL}$ of $80 \%$ methanol. The eluate was collected, dried overnight in a Speed-Vac ${ }^{\circ}$ Plus SC210A concentrator, and residue resolubilized in $5 \mathrm{~mL}$ of DI water.

\subsubsection{Identification of blackberry phenolics}

Aliquots $(5 \mathrm{~mL})$ of phenolic extracts obtained by the previous solvent extraction were dried using a Speed-Vac $($ Plus SC210A concentrator and brought back to a volume of $1 \mathrm{~mL}$ in $5 \%$ formic acid for anthocyanins analysis and 50\% methanol for flavonols analysis. The solutions were then filtered through a $0.45 \mu \mathrm{m}$ filter (Whatman, Marlborough, MA) prior to High Performance Liquid 
Chromatography (HPLC) and High Performance Liquid Chromatography Mass Spectrometry (HPLC-MS) analysis.

Anthocyanins were identified according to the HPLC-MS method described by Cho et al. (2004). The HPLC system consisted of a Hewlett Packard 1100 HPLC (Hewlett Packard, Palo Alto, CA) equipped with an autosampler, binary HPLC pump and UV/VIS detector. A $250 \times 4.60 \mathrm{~mm}$ Symmetry $5 \mu \mathrm{m} \mathrm{C}_{18}$ column (Waters Corp., Milford, MA) was used for separation with compounds monitored at $510 \mathrm{~nm}$. The mobile phase consisted of a $5 \%$ formic acid solution (solvent A) and methanol (solvent B). At a solvent flow rate of $1 \mathrm{~mL} / \mathrm{min}$, the concentration of solvent A varied from $98 \%$ to $40 \%$ from $0-60 \mathrm{~min}$, and then to $98 \%$ from $61-85$ min. The system was interfaced to a Bruker Esquire HPLC-MS ion trap mass spectrophotometer with the following conditions: positive ion mode, capillary voltage of $4,000 \mathrm{~V}$, nebulizing pressure of $30 \mathrm{psi}$, drying gas flow of $9 \mathrm{~mL} / \mathrm{min}, 300{ }^{\circ} \mathrm{C}$, and a full scan mode comprising a mass range of $m / z 50$ to 1,000 at $1 \mathrm{~s} / \mathrm{cycle}$. Compounds were identified using the $\mathrm{m} / \mathrm{z}$ data, retention time and previous identifications reported by Cho et al. (2004).

Flavonols were identified according to the HPLC-MS method described by Cho et al. (2005) using the same HPLC-MS system described above, except peaks were monitored at $360 \mathrm{~nm}$ and a 250 $\times 4.60 \mathrm{~mm}$ Aqua ${ }^{\circledR} \mathrm{C}_{18}$ column (Phenomenex, Torrance, CA) was used for separation. The mobile phase consisted of a $2 \%$ aqueous solution of acetic acid (solvent A) and $0.5 \%$ acetic acid in water and acetonitrile at a ratio of $1: 1(\mathrm{v} / \mathrm{v}$, solvent $\mathrm{B})$. Using a solvent flow rate of $1 \mathrm{~mL} / \mathrm{min}$, the concentration of solvent A started at $90 \%$ from 0-50 min, then decreased to $45 \%$ from 51-60 min and ended at $90 \%$ from $61-75 \mathrm{~min}$. The system was interfaced to a Bruker Esquire HPLC-MS ion trap mass spectrophotometer with the following conditions: negative ion mode, capillary voltage of $4,000 \mathrm{~V}$, nebulizing pressure of $30 \mathrm{psi}$, drying gas flow of $9 \mathrm{~mL} / \mathrm{min}, 300{ }^{\circ} \mathrm{C}$, and a full scan mode comprising a mass range of $m / z 50$ to 1,000 at $1 \mathrm{~s} / \mathrm{cycle}$. Compounds were identified using the $\mathrm{m} / \mathrm{z}$ data, retention times and previous identifications reported by Cho et al. (2005).

\subsubsection{Quantification of blackberry phenolics}

Anthocyanins were quantified by injecting $50 \mu \mathrm{L}$ of filtered phenolic extract into a Waters HPLC system (Milford, MA) equipped with a model 600 pump, model 717 autosampler and model 996 photodiode array detector. The same HPLC conditions described above were used. External calibration curves of a cyanidin 3-glucoside (C3G) standard (10-100 $\mu \mathrm{g} / \mathrm{mL}$, Polyphenol AS, Norway) were used to quantify anthocyanins, with results expressed as $\mu \mathrm{g}$ of $\mathrm{C} 3 \mathrm{G}$ equivalents $(\mathrm{C} 3 \mathrm{GE})$ per $\mathrm{mL}$ extract.

Flavonols were quantified by injecting $50 \mu \mathrm{L}$ of filtered phenolic extract into the same Waters HPLC system described above for quantification of anthocyanins and the HPLC-MS conditions described above for identification of flavonols. External calibration curves of a rutin standard $(10-100 \mu \mathrm{g} / \mathrm{mL}$, Millipore Sigma, St. Louis, MO) were used to quantify flavonols, with results expressed as $\mu \mathrm{g}$ of rutin equivalents (RE) per $\mathrm{mL}$ extract.

\subsubsection{Low temperature vacuum distillation of blackberry vola- tiles}

For each genotype, $150 \mathrm{~g}$ of fresh blackberries, $150 \mathrm{~mL}$ of deionized water and $50 \mathrm{~g}$ of $\mathrm{NaCl}$ were blended and then vacuum distilled for $30 \mathrm{~min}$ at $28 \mathrm{in}$. $\mathrm{Hg}$ in a $50{ }^{\circ} \mathrm{C}$ water bath, $0{ }^{\circ} \mathrm{C}$ condenser using a Buchi Rotavapor R-114 (Buchi, Flawil, Switzerland). The first $100 \mathrm{~mL}$ were collected in a flask stored in an ice water bath and frozen at $-20{ }^{\circ} \mathrm{C}$ until use. The extraction was repeated three times for each genotype.

In order to verify that no phenolic compounds were present in the volatile extracts, they were analyzed by HPLC following the protocol described above, except the detection wavelength was $280 \mathrm{~nm}$.

\subsubsection{Identification and quantification of blackberry volatiles}

Two samples $(4 \mathrm{~mL})$ of low temperature vacuum distillates from each genotype were placed in vials and preheated to $65^{\circ} \mathrm{C}$ before a $85 \mu \mathrm{m} \mathrm{DVB/CAR/PDMS} \mathrm{Solid} \mathrm{Phase} \mathrm{Microextraction} \mathrm{(SPME)}$ fiber was placed in the headspace above the sample. The vials were held for $30 \mathrm{~min}$ on a stir plate set at $65^{\circ} \mathrm{C}$ before removing the SPME fiber and GC injection.

Gas chromatography analysis was performed using a Shimadzu GC-2010 Plus Gas Chromatograph equipped with a Flame Ionization Detector (GC-FID) and a GCMS-QP2010 SE Mass Spectrometer (GC-MS). Samples were analyzed by both GC-FID and GC-MS and separation was performed on each using a HP-5 (30 $\mathrm{m} \times 0.25 \mathrm{~mm}$ inner diameter, $5 \%$ phenyl-methylpolysiloxane, 1.0 $\mu \mathrm{m}$ film thickness) capillary column (Agilent, Santa Clara, CA). For both GC-MS and GC-FID analysis, the injector temperature was $250{ }^{\circ} \mathrm{C}$. Helium was used as the carrier gas and column flow rate was $1.92 \mathrm{~mL} / \mathrm{min}$ for GC-FID and $1.20 \mathrm{~mL} / \mathrm{min}$ for GC-MS. The oven temperature was programmed for a 4 -min hold at $30^{\circ} \mathrm{C}$, then $30{ }^{\circ} \mathrm{C}$ to $180{ }^{\circ} \mathrm{C}$ at $6{ }^{\circ} \mathrm{C} / \mathrm{min}$, then from $180{ }^{\circ} \mathrm{C}$ to $280{ }^{\circ} \mathrm{C}$ at $8^{\circ} \mathrm{C} / \mathrm{min}$, and a final $3 \mathrm{~min}$ hold at $280^{\circ} \mathrm{C}$. The GC-FID detector temperature was $280^{\circ} \mathrm{C}$ and the interface temperature for the GCMS had an ion source temperature of $230^{\circ} \mathrm{C}$ and an interface temperature of $250^{\circ} \mathrm{C}$. GC-MS was performed in full scan mode, with a scan range of $20-300 \mathrm{~m} / \mathrm{z}$. The volatiles were identified by comparison of their mass spectra with the National Institute of Standards and Technology NIST17 spectral library, literature data, and retention indices. The retention indices were performed after running C5-C20 alkane standards (Millipore Sigma, St. Louis, MO) and online searches of similar work with HP5 or DB5 columns. Individual compounds were quantified using external calibration curves of authentic standards. Compounds with no standard available were quantified using calibration curves of a compound of similar class that eluted in close proximity. The standards used to quantify each compound are listed in Table 1.

\subsubsection{Total phenolic assessment}

The total phenolic content of blackberry phenolic and volatile extracts was assessed using the Folin-Ciocalteu assay (Slinkard and Singleton, 1977). Phenolic extracts were diluted 50-fold and volatile extracts were not diluted. The absorbance was read at $760 \mathrm{~nm}$, with results expressed as $\mu \mathrm{g}$ of Gallic Acid Equivalents (GAE) per $\mathrm{mL}$ of extract.

\subsubsection{Antioxidant capacity assessment}

The antioxidant capacity of blackberry phenolic and volatile extracts was assessed by the 2,2-diphenyl-1-picrylhydrazyl (DPPH) assay (Gorinstein et al, 2004). A standard solution of $1,000 \mu \mathrm{M}$ Trolox was diluted to obtain a range of concentrations from 31.25 to $1,000 \mu \mathrm{M}$. A volume of $10 \mu \mathrm{L}$ of Trolox dilutions, methanol blank, non-diluted blackberry essence, and 50-fold diluted phenolic extracts was added in a 96-well microplate in triplicate. Then, a 
Table 1. Volatile composition $(\mathrm{ng} / \mathrm{mL}$ ) of low temperature vacuum distillates obtained from three blackberry genotypes

\begin{tabular}{|c|c|c|c|c|c|}
\hline \multirow{2}{*}{ Compound } & \multirow{2}{*}{ Quantified as } & \multirow{2}{*}{$\mathbf{R} \mathbf{I}^{1}$} & \multicolumn{3}{|c|}{ Genotype } \\
\hline & & & A2528T & A2587T & Natchez \\
\hline 1-Butanol & 1-Butanol & 642 & ND & $1.53 \pm 0.62^{2} a^{3}$ & $6.53 \pm 4.16 a$ \\
\hline 2-Methylbutan-1-ol & 1-Butanol, 2-methyl- & 723 & $0.71 \pm 0.12 a$ & ND & $0.92 \pm 0.28 a$ \\
\hline Trans-3-hexen-1-ol & Trans-3-hexen-1-ol & 846 & $1.79 \pm 0.13 a$ & $0.31 \pm 0.08 b$ & $0.79 \pm 0.13 c$ \\
\hline Cis-3-hexen-1-ol & Cis-3-hexen-1-ol & 851 & $8.90 \pm 0.94 a$ & $5.73 \pm 0.88 b$ & $9.07 \pm 1.25 a$ \\
\hline 2-hexen-1-ol, & 1-Hexanol & 861 & $16.89 \pm 1.73 a$ & $1.85 \pm 0.36 b$ & $2.94 \pm 1.08 b$ \\
\hline 1-Hexanol & 1-Hexanol & 863 & $26.36 \pm 1.34 a$ & $5.56 \pm 0.57 b$ & $34.68 \pm 4.83 c$ \\
\hline 2-Heptanol & 2-Heptanol & 895 & $8.92 \pm 1.32 b$ & $190.43 \pm 28.11 \mathrm{a}$ & $1.68 \pm 0.30 b$ \\
\hline 1-Heptanol & 1-Heptanol & 968 & $1.19 \pm 0.49 a$ & $1.25 \pm 0.29 a$ & $0.83 \pm 0.22 a$ \\
\hline 1-Octen-3-ol & 1-Octen-3-ol & 978 & $0.94 \pm 0.18 a$ & $1.36 \pm 0.69 a$ & $0.76 \pm 0.05 a$ \\
\hline 1-Pentanol, 3-ethyl-4-methyl- & 1-Hexanol & 1,019 & $0.21 \pm 0.09$ & ND & ND \\
\hline 2-Ethyl-1-hexanol & 1-Hexanol & 1,028 & $3.74 \pm 0.55 a$ & $3.17 \pm 1.31 \mathrm{a}$ & $3.96 \pm 0.64 a$ \\
\hline 2- Phenylethyl alcohol & Phenylethyl alcohol & 1,117 & ND & $44.21 \pm 2.78 a$ & $23.47 \pm 3.74 b$ \\
\hline 1-Nonanol & 1-Nonanol & 1,169 & $0.82 \pm 0.24 b$ & $2.56 \pm 1.01 a$ & $1.09 \pm 0.17 b$ \\
\hline 6-Camphenol & $\alpha$-Terpineol & 1,177 & $2.49 \pm 0.93 b$ & ND & $40.42 \pm 3.75 a$ \\
\hline 1-Decanol & 1-Decanol & 1,271 & $0.36 \pm 0.19 b$ & $1.45 \pm 0.52 a$ & $0.69 \pm 0.08 b$ \\
\hline p-Mentha-1-en-9-ol & Perillic alcohol & 1,305 & ND & $32.72 \pm 2.98 a$ & $10.71 \pm 1.36 b$ \\
\hline 1-Undecanol & 1-Decanol & 1,371 & $0.05 \pm 0.09$ & ND & ND \\
\hline Dodecanol & 1-Decanol & 1,474 & $0.67 \pm 0.30 b$ & $1.65 \pm 0.13 a$ & $0.28 \pm 0.15 b$ \\
\hline Total alcohols & & & $74.04 \pm 7.71 b$ & $293.79 \pm 40.33 a$ & $138.84 \pm 18.45 b$ \\
\hline 2-Butenal (E) & Butanal, 3-methyl- & 629 & $0.37 \pm 0.33$ & ND & ND \\
\hline 2-Butenal (Z) & Butanal, 3-methyl- & 647 & $9.58 \pm 2.01$ & ND & ND \\
\hline Pentanal & Pentanal & 677 & $4.32 \pm 2.43 a$ & $3.36 \pm 1.60 a$ & $1.46 \pm 0.27 a$ \\
\hline 2-Ethyl-2-butenal & Butanal, 3-methyl- & 727 & $1.06 \pm 0.20 a$ & ND & $2.34 \pm 0.53 b$ \\
\hline 2-Pentenal & Pentanal & 744 & $1.30 \pm 0.60 a$ & $0.31 \pm 0.27 b$ & $0.33 \pm 0.29 b$ \\
\hline 3-Methyl-2-butenal & Butanal, 3-methyl- & 776 & $0.63 \pm 0.64 a$ & $1.13 \pm 0.38 a$ & $0.82 \pm 0.19 a$ \\
\hline Hexanal & Hexanal & 798 & $3.46 \pm 0.43 a$ & $4.20 \pm 1.23 a$ & $4.08 \pm 0.68 a$ \\
\hline Heptanal & Heptanal & 902 & $0.05 \pm 0.01 a$ & $0.09 \pm 0.03 a$ & $0.06 \pm 0.02 a$ \\
\hline Benzaldehyde & Benzaldehyde & 965 & $2.20 \pm 0.51 b$ & $1.57 \pm 0.03 b$ & $4.85 \pm 1.13 a$ \\
\hline Octanal & Octanal & 1,003 & $1.64 \pm 0.35 b$ & $4.30 \pm 2.04 a$ & $1.31 \pm 0.50 b$ \\
\hline Nonanal & Nonanal & 1,104 & $4.48 \pm 4.18 a$ & $8.48 \pm 3.74 a$ & $2.26 \pm 0.47 a$ \\
\hline Decanal & Decanal & 1,206 & $2.85 \pm 1.02 a$ & $9.08 \pm 5.78 a$ & $7.02 \pm 1.30 a$ \\
\hline 2-Decenal & 2-Decenal & 1,265 & $0.71 \pm 0.41 b$ & $1.35 \pm 0.75 b$ & $2.55 \pm 0.43 a$ \\
\hline Perilla aldehyde & 2-Decenal & 1,289 & ND & $0.67 \pm 0.10$ & ND \\
\hline Undecanal & Undecenal & 1,308 & $0.29 \pm 0.16 a$ & $0.87 \pm 0.59 a$ & ND \\
\hline Dodecanal & Decanal & 1,411 & $0.34 \pm 0.21 \mathrm{ab}$ & $0.63 \pm 0.24 a$ & $0.14 \pm 0.09 b$ \\
\hline Total aldehydes & & & $33.85 \pm 13.80 a$ & $36.04 \pm 16.78 a$ & $27.22 \pm 5.89 a$ \\
\hline$\alpha, p$-Dimethylstyrene & $\alpha, p$-Dimethylstyrene & 1,095 & $9.80 \pm 5.23 a$ & $45.74 \pm 6.70 b$ & $27.83 \pm 3.63 c$ \\
\hline Total aromatic hydrocarbons & & & $9.80 \pm 5.23 c$ & $45.74 \pm 6.70 a$ & $27.83 \pm 3.63 b$ \\
\hline Ethyl acetate & Ethyl acetate & 593 & $76.29 \pm 11.89 a$ & $50.07 \pm 38.26 a$ & $67.25 \pm 13.08 a$ \\
\hline Methyl propionate & Methyl butanoate & 604 & ND & $0.23 \pm 0.21$ & ND \\
\hline Ethyl propanoate & Methyl butanoate & 699 & $0.26 \pm 0.18 a$ & $0.25 \pm 0.12 a$ & ND \\
\hline Methyl butanoate & Methyl butanoate & 711 & $0.30 \pm 0.19 a$ & $0.25 \pm 0.09 a$ & ND \\
\hline Methyl 3-methylbutanoate & Methyl butanoate & 770 & $0.19 \pm 0.21$ & ND & ND \\
\hline Ethyl butanoate & Ethyl butanoate & 794 & $1.70 \pm 0.35$ & ND & ND \\
\hline
\end{tabular}


Table 1. Volatile composition $(\mathrm{ng} / \mathrm{mL}$ ) of low temperature vacuum distillates obtained from three blackberry genotypes - (continued)

\begin{tabular}{|c|c|c|c|c|c|}
\hline \multirow{2}{*}{ Compound } & \multirow{2}{*}{ Quantified as } & \multirow{2}{*}{$\mathbf{R} \mathbf{I}^{1}$} & \multicolumn{3}{|c|}{ Genotype } \\
\hline & & & A2528T & A2587T & Natchez \\
\hline Butyl acetate & Butyl acetate & 815 & $0.84 \pm 0.27$ & ND & ND \\
\hline Methyl hexanoate & Methyl hexanoate & 921 & $1.38 \pm 1.01 a$ & $0.33 \pm 0.25 a$ & $0.57 \pm 0.18 a$ \\
\hline Hexyl formate & Methyl butanoate & 929 & $0.27 \pm 0.11$ & ND & ND \\
\hline Ethyl 3-hydroxybutyrate & Methyl butanoate & 939 & ND & ND & $1.05 \pm 0.17$ \\
\hline Ethyl hexanoate & Ethyl hexanoate & 998 & $2.36 \pm 1.44 a$ & $1.56 \pm 0.49 a$ & $2.47 \pm 0.36 a$ \\
\hline Methyl octanoate & Methyl octanoate & 1,122 & $0.44 \pm 0.21 a$ & $0.36 \pm 0.20 a$ & $0.22 \pm 0.11 a$ \\
\hline Total esters & & & $84.05 \pm 15.85 a$ & $53.06 \pm 39.61 a$ & $71.56 \pm 13.89 a$ \\
\hline 2-Heptanone & 2-Heptanone & 890 & $0.88 \pm 0.35 a b$ & $0.84 \pm 0.04 a$ & $1.37 \pm 0.24 b$ \\
\hline 5-Hepten-2-one, 6-methyl- & 5-Hepten-2-one, 6-methyl- & 987 & $0.50 \pm 0.11 a$ & $0.52 \pm 0.14 a$ & $0.23 \pm 0.03 b$ \\
\hline Acetophenone & Acetophenone & 1,076 & ND & $0.73 \pm 0.68 a$ & $0.71 \pm 1.23 a$ \\
\hline Total ketones & & & $1.38 \pm 0.46 a$ & $2.09 \pm 0.86 a$ & $2.31 \pm 1.50 a$ \\
\hline$\beta$-Myrcene & $\beta$-Myrcene & 992 & $4.25 \pm 0.92 a$ & $14.21 \pm 1.01 b$ & $6.82 \pm 0.52 c$ \\
\hline D-Limonene & D-Limonene & 1,036 & $2.05 \pm 0.82 a$ & $10.50 \pm 1.87 b$ & $6.96 \pm 1.67 c$ \\
\hline p-Cymene & $\mathrm{p}$-Cymene & 1,040 & $0.22 \pm 0.10 a$ & $2.60 \pm 0.41 b$ & $1.04 \pm 0.003 c$ \\
\hline m-Cymene & $\mathrm{p}$-Cymene & 1,045 & ND & ND & ND \\
\hline$\beta$-Ocimene & $\beta$-Myrcene & 1,050 & $0.56 \pm 0.15 b$ & $2.74 \pm 0.54 a$ & $1.13 \pm 0.17 b$ \\
\hline Dihydro myrcenol & Myrtenol & 1,072 & $0.52 \pm 0.11 a$ & $0.47 \pm 0.35 a$ & $0.14 \pm 0.07 a$ \\
\hline Linalool oxide & Linalool & 1,078 & $0.48 \pm 0.11$ & ND & ND \\
\hline Linalool & Linalool & 1,100 & $5.09 \pm 1.30 b$ & $11.59 \pm 0.97 a$ & $4.47 \pm 0.29 b$ \\
\hline 6-Camphenone & Acetophenone & 1,130 & $0.47 \pm 0.07 a$ & ND & $2.36 \pm 0.11 b$ \\
\hline Cis-p-mentha-2,8-dien-1-ol & 1-Nonanol & 1,142 & ND & $0.56 \pm 0.20$ & ND \\
\hline Verbenol & Terpinen-4-ol & 1,158 & ND & $0.88 \pm 0.16 a$ & $2.53 \pm 1.16 a$ \\
\hline Isopulegol & Isopulegol & 1,164 & $0.37 \pm 0.12$ & ND & ND \\
\hline$\alpha$-Phellandrene-8-ol & $\alpha$-Terpineol & 1,175 & ND & $29.54 \pm 6.83$ & ND \\
\hline Borneol & Borneol & 1,187 & $0.96 \pm 0.26 a$ & $4.03 \pm 0.50 b$ & $7.45 \pm 0.79 c$ \\
\hline Terpinen-4-ol & Terpinen-4-ol & 1,188 & $4.91 \pm 2.67 a$ & $52.89 \pm 4.20 b$ & $35.44 \pm 4.86 c$ \\
\hline p-Cymene-8-ol & Terpinen-4-ol & 1,191 & $5.78 \pm 3.21 a$ & $32.37 \pm 5.65 b$ & $19.75 \pm 4.94 c$ \\
\hline$\alpha$-Terpineol & $\alpha$-Terpineol & 1,200 & $24.55 \pm 13.61 b$ & $68.60 \pm 13.80 a$ & $36.21 \pm 4.45 b$ \\
\hline Myrtenol & Myrtenol & 1,208 & $1.67 \pm 0.64 b$ & $18.58 \pm 6.97 a$ & $1.88 \pm 0.46 b$ \\
\hline Nerol & Nerol & 1,224 & $5.80 \pm 2.31 a$ & $7.20 \pm 1.02 a$ & $5.12 \pm 0.92 a$ \\
\hline Verbenone & D-Carvone & 1,228 & $2.54 \pm 1.11 b$ & $35.07 \pm 5.02 a$ & $7.67 \pm 1.37 b$ \\
\hline Citronellol & Citronellol & 1,233 & $0.42 \pm 0.28 a$ & $6.32 \pm 0.86 b$ & $4.81 \pm 0.78 c$ \\
\hline D-Carvone & D-Carvone & 1,256 & ND & $51.87 \pm 8.33$ & ND \\
\hline Geraniol & Geraniol & 1,258 & $5.59 \pm 1.36 b$ & $5.02 \pm 0.67 b$ & $16.19 \pm 3.86 a$ \\
\hline Geranial & 2-Decenal & 1,274 & $0.53 \pm 0.31 b$ & $1.97 \pm 0.42 a$ & $0.88 \pm 0.22 b$ \\
\hline Perillic alcohol & Perillic alcohol & 1,299 & $1.87 \pm 1.22 \mathrm{a}$ & $14.38 \pm 1.03 b$ & $7.30 \pm 1.47 c$ \\
\hline$\beta$-Damascenone & $\beta$-Damascenone & 1,398 & $11.08 \pm 6.85 a$ & $21.85 \pm 1.85 a$ & ND \\
\hline Total monoterpenes & & & $79.72 \pm 36.52 c$ & $393.23 \pm 61.23 a$ & $168.15 \pm 27.58 b$ \\
\hline$\beta$-Ionone & $\beta$-Ionone & 1,502 & $0.42 \pm 0.16 a$ & $0.26 \pm 0.01 \mathrm{ab}$ & $0.10 \pm 0.01 b$ \\
\hline Total Noisoprenoids & & & $0.42 \pm 0.16 b$ & $0.26 \pm 0.01 a$ & $0.10 \pm 0.01 b$ \\
\hline Epicubenol & Perillic alcohol & 1,657 & ND & $19.73 \pm 3.45 a$ & $5.23 \pm 1.56 b$ \\
\hline$\alpha$-Cadinol & Perillic alcohol & 1,669 & ND & $7.78 \pm 3.78 a$ & $2.39 \pm 0.83 a$ \\
\hline Total sesquiterpenes & & & $0.42 \pm 0.16 b$ & $27.77 \pm 7.24 a$ & $7.72 \pm 2.40 b$ \\
\hline Total volatiles & & & $283.25 \pm 45.39 c$ & $851.73 \pm 49.23 a$ & $443.62 \pm 34.98 b$ \\
\hline
\end{tabular}

${ }^{1} \mathrm{RI}=$ retention index. ${ }^{2}$ Means $\left(\mathrm{N}=3 \pm \mathrm{Std}\right.$.Dev.). ${ }^{3}$ Means within rows with different letters are significantly different $(\mathrm{p}<0.05)$. 
Table 2. Standard volatiles used to prepare the simulated blackberry volatile mixture

\begin{tabular}{|c|c|c|c|}
\hline Compound & Concentration $(\mathrm{ng} / \mathrm{mL}$ ) & Compound & Concentration $(\mathrm{ng} / \mathrm{mL})$ \\
\hline 2-Heptanol & 67.01 & Hexanal & 3.91 \\
\hline Ethyl acetate & 64.54 & Citronellol & 3.85 \\
\hline$\alpha$-Terpineol & 43.12 & Pentanal & 3.04 \\
\hline Terpinen-4-ol & 31.08 & Benzaldehyde & 2.87 \\
\hline$\alpha, p$-Dimethylstyrene & 27.79 & 1-Butanol, 3-methyl- & 2.59 \\
\hline 1-Octanol & 22.30 & Octanal & 2.42 \\
\hline 1-Hexanol & 22.20 & Ethyl hexanoate & 2.13 \\
\hline Geraniol & 8.93 & Toluene & 1.68 \\
\hline$\beta$-Myrcene & 8.43 & 2-Decenal & 1.54 \\
\hline 3-Hexen-1-ol & 7.90 & 1-Nonanol & 1.49 \\
\hline Perillic alcohol & 7.85 & Cymene & 1.29 \\
\hline Myrtenol & 7.38 & 1-Heptanol & 1.09 \\
\hline Linalool & 7.05 & 2-Heptanone & 1.03 \\
\hline Thymol & 6.59 & 1-Octen-3-ol & 1.02 \\
\hline$D$-Limonene & 6.50 & 3-Hexen-1-ol & 0.97 \\
\hline Decanal & 6.32 & 1-Decanol & 0.83 \\
\hline 2-Hexenal & 6.25 & 5-Hepten-2-one, 6-methyl- & 0.42 \\
\hline Nerol & 6.04 & Methyl octanoate & 0.34 \\
\hline Nonanal & 5.07 & $\beta$-Ionone & 0.26 \\
\hline$\alpha$-Terpinene & 5.00 & 2-Octenal & 0.22 \\
\hline 1-Pentanol & 4.32 & Heptanal & 0.07 \\
\hline Borneol & 4.15 & Total concentration & 407 \\
\hline
\end{tabular}

volume of $140 \mu \mathrm{L}$ of $0.1 \mathrm{mM}$ DPPH was added to each well. The plate was incubated in the dark for $30 \mathrm{~min}$ before reading the absorbance at $517 \mathrm{~nm}$. Results are expressed as $\mu \mathrm{M}$ of Trolox equivalents (TE) per $\mathrm{mL}$ of extract.

\subsubsection{Preparation of the standard volatiles mix}

In order to confirm that the anti-inflammatory effect observed within the blackberry volatile extracts was due to the volatiles and not other compounds co-extracted via the hydrodistillation process, a mix of standard volatiles was prepared based on the list of volatiles previously identified in the blackberry volatile extracts obtained by low temperature vacuum distillation. Only one solution was prepared, and the concentration of each volatile standard was based on the average concentration among the three genotypes, which resulted in a list of 43 volatiles (Table 2).

\subsection{Cell culture conditions and treatments}

\subsubsection{Cell culture conditions}

RAW 264.7 murine macrophage cells were purchased from the American Type Culture Collection (ATCC, Manassas, VA) and cultured in Dulbecco's modified eagle's medium (DMEM) en- riched with $1 \%$ penicillin-streptomycin, $1 \% \mathrm{~L}$-glutamine and $10 \%$ fetal bovine serum (FBS). The cells were incubated at $37{ }^{\circ} \mathrm{C}$ in a $5 \% \mathrm{CO}_{2}$ environment in $75 \mathrm{~cm}^{2}$ culture flasks. All reagents used for cell culture were purchased from Promega ${ }^{\circledR}$ (Madison, WI) and Thermo Fischer Scientific (Waltham, MA).

\subsubsection{Cell culture treatments}

For each genotype, three replicates of phenolic extracts were pooled, as well as the three replicates of volatile extracts. Targeting a cell density of $1.0 \times 10^{4}$ cells/well, $0.5 \mathrm{~mL}$ of RAW264.7 cells in DMEM media were seeded in a 24 -well plate and incubated at $37{ }^{\circ} \mathrm{C}$ and $5 \% \mathrm{CO}_{2}$ for $24 \mathrm{hr}$. After incubation, the media was replaced by fresh media containing $0.02 \%$ of Tween 80 and either a 2 -fold, 4 -fold or 8 -fold dilution of volatile extract, or 10-fold, 20-fold or 40-fold dilution of phenolic extract. The positive (i.e. LPS-induced cells, no treatment) and negative (i.e. no inflammation, no treatment) controls were incubated with working media containing $0.02 \%$ Tween 80 . After incubating for one $\mathrm{hr}$, the treatment was replaced by media containing $100 \mathrm{ng}$ lipopolysaccharides (LPS)/mL for $24 \mathrm{hr}$, except in the case of the negative control in which the media was replaced by fresh working media. Then, the supernatants were centrifuged for $1 \mathrm{~min}$ at $140 \mathrm{~g}$, aliquoted and frozen at $-80^{\circ} \mathrm{C}$ until all samples required for the next step were collected. The experiment was repeated over three wells per treatment, and three times per genotype, each time using a different set of RAW264.7 cells. 
Table 3. Composition and antioxidant activity of blackberry phenolic extracts

\begin{tabular}{|c|c|c|c|}
\hline \multirow{2}{*}{ Compound } & \multicolumn{3}{|c|}{ Genotype } \\
\hline & A2528T & A2587T & Natchez \\
\hline Total anthocyanins ${ }^{1}$ ( $\mu \mathrm{g}$ C3GE/mL) & $1,484 \pm 96 b^{4,5}$ & $2,078 \pm 28 a$ & $2,263 \pm 144 a$ \\
\hline Total flavonols ${ }^{1}$ ( $\mu \mathrm{g} R E / m L$ ) & $163 \pm 13 a$ & $105 \pm 16 b$ & $129 \pm 13 a b$ \\
\hline Total phenolics ${ }^{2}$ ( $\mu \mathrm{g}$ GAE$/ \mathrm{mL}$ ) & $4,315 \pm 121 \mathrm{a}$ & $3,369 \pm 92 b$ & $3,680 \pm 171 \mathrm{c}$ \\
\hline Antioxidant activity ${ }^{3}(\mu \mathrm{mol} \mathrm{TE} / \mathrm{mL})$ & $30 \pm 1 a$ & $24 \pm 1 b$ & $25 \pm 2 b$ \\
\hline
\end{tabular}

${ }^{1}$ Total anthocyanins and total flavonols were measured by HPLC-PDA and expressed as cyanidin 3-glucoside equivalents (C3GE) and rutin equivalents (RE), respectively. ${ }^{2}$ Total phenolics were measured using the Folin Ciocalteu assay and are expressed as gallic acid equivalents (GAE). ${ }^{3}$ Antioxidant activity was measured by the DPPH method and expressed as Trolox equivalents (TE). ${ }^{4}$ Results are expressed as mean $\pm S D(n=3) .{ }^{5}$ Means within rows with different letters are significantly different $(p<0.05)$.

\subsubsection{Measurement of cell viability}

The effect of phenolic and volatile extracts on cell viability was measured by the MTS assay using a CellTiter 96 Aqueous One Solution Cell Proliferation Assay (Promega Co., Madison, WI). Cells were seeded in three different 96-well plate and incubated for $24 \mathrm{hrs}$. Then, cells were treated with a 10-, 20-, or 40-fold diluted phenolic extract or a 2-, 4-, or 8-fold diluted volatile extract. After an hr of exposure to the extracts, each well was rinsed twice with working media before adding a volume of 100 $\mu \mathrm{L}$ of working media and $20 \mu \mathrm{L}$ of MTS reagent in each well. The plate was incubated for one hr before reading the absorbance of wells at $490 \mathrm{~nm}$. The manipulation was repeated after the second and third hr of exposure to the blackberry extracts with the remaining plates. The concentration in formazan obtained by absorbance measurement was directly proportional to the number of living cells. Consequently, the viability of cells within one, two and three hrs of exposition with the blackberry extracts was calculated as a percentage with a non-treated control as reference.

\subsubsection{Measurement of nitric oxide}

The Griess Reagent System was used to measure NO production as a marker of inflammation. Using a 96-well plate, a nitrite standard reference curve was obtained through serial dilutions leading to a concentration range of 0 to $100 \mu \mathrm{M}$. Then, $50 \mu \mathrm{L}$ of sulfanilamide solution was added to $50 \mu \mathrm{L}$ of each supernatant and incubated for $10 \mathrm{~min}$ in the dark, followed by $50 \mu \mathrm{L}$ of $\mathrm{N}$-1-napthylethylenediamine dihydrochloride (NED) for $10 \mathrm{~min}$ in the dark. Finally, the absorbance was read by the plate reader at $540 \mathrm{~nm}$, and the absorbance values were converted into NO levels using the nitrite standard curve and after deducting the background noise absorbance measured by the sample control media.

\subsubsection{Measurement of IL- 6 and TNF- $\alpha$}

Enzyme-linked immunosorbent assays (ELISA) kits were used to measure levels of IL-6 and TNF- $\alpha$ (mouse IL-6 ELISA and mouse TNF- $\alpha$ ELISA, RayBiotech Inc., Norcross, GA). Prior to testing, cell culture supernatants were diluted 20 -fold. The measurements were then performed according to the manufacturer's instruction.

\subsection{Experimental design and statistical methods}

Significant differences in total phenolics, anthocyanins, fla- vonols, volatiles and DPPH among genotypes were assessed using Tukey's HSD test with a critical value of $5 \%$. Significant differences in NO, IL-6 or TNF- $\alpha$ concentrations between the controls and phenolic or volatile extract treatments were assessed through a nested ANOVA with pairwise differences determined using Tukey's HSD multiple comparison adjustments. A variable "plate_ID" was attributed to each replicate based on the plate it was tested on. The samples tested on one specific plate had the same cell line batch and cell passage, which made them dependent. To correct for this condition, a random effect was applied to the model based on the plate ID variable. Differences between means were determined by Tukey's HSD test. The critical value of all tests was $5 \%$.

\section{Results and discussion}

\subsection{Blackberry phenolic composition}

The composition of the phenolic extracts of A2528T, A2587T and Natchez are presented in Table 3. The total phenolic concentrations were 4,315 $\pm 121 \mu \mathrm{g} / \mathrm{mL}$ (GAE) for A2528T, 3,369 $\pm 92 \mu \mathrm{g} / \mathrm{mL}$ for $\mathrm{A} 2587 \mathrm{~T}$, and 3,680 $\pm 171 \mu \mathrm{g} / \mathrm{mL}$ for Natchez, which fell within concentration ranges $(1,140-10,560 \mu \mathrm{g} / \mathrm{mL})$ reported in previous studies (Kaume et al., 2012).

The typical blackberry anthocyanins, i.e. cyanidin 3-glucoside, cyanidin 3-rutinoside, cyanidin 3-xyloside, cyanidin 3-malonylglucoside and cyanidin 3-dioxalylglycoside dioxalylglycoside (Cho et al., 2004, Kaume et al., 2012, Wu and Prior, 2005) were detected in all genotypes, except Natchez in which cyanidin 3-xyloside was not detected (Table s1). The ranges of concentration in anthocyanins varied between 1,484 \pm 96 and 2,263 $\pm 144 \mu \mathrm{g} /$ $\mathrm{mL}$ (fresh weight) $\mathrm{C} 3 \mathrm{G}$ equivalents, which was in accordance with previous studies (Cho et al., 2004, Kaume et al., 2012, Wu and Prior, 2005).

A total of six flavonols were identified in A2528T, seven in A2587T and nine in Natchez (Table s2), leading to total flavonol concentrations of $163 \pm 13,105 \pm 16$, and $129 \pm 13 \mu \mathrm{g} / \mathrm{mL}$ (fresh weight) RE, for A2528T, A2587T and Natchez, respectively. These profiles and concentrations were consistent with previous studies (Kaume et al., 2012, Cho et al., 2005, Kolniak-Ostek et al., 2015, Mikulic-Petkovsek et al., 2012).

\subsection{Blackberry volatile composition}

The composition of the volatile extracts of A2528T, A2587T and Natchez obtained by low temperature vacuum distillation is pre- 


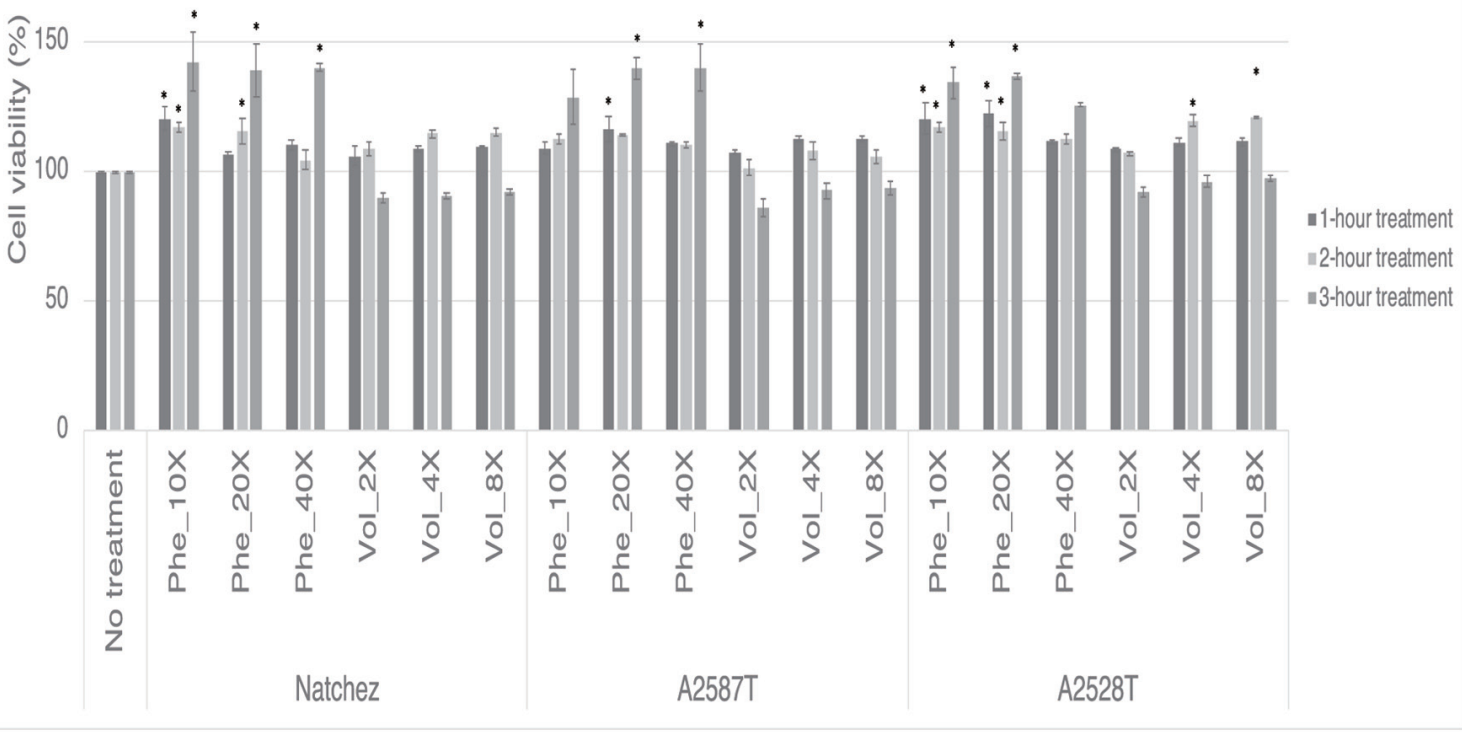

Figure 1. Percentage of viability of RAW264.7 cells after a preventive treatment of one, two and three hrs with phenolic extracts ("Phe") diluted 10-, 20-, or 40-fold or volatile extracts ("Vol”) diluted 2-, 4-, or 8-fold. Values represent mean \pm SEM $(n=3)$. * Denotes significant difference from cells that received no treatment $(\mathrm{P}<0.05)$.

sented in Table 1 . The concentrations of total volatiles were 283 $\pm 45 \mathrm{ng} / \mathrm{mL}$ for A2528T, $852 \pm 49 \mathrm{ng} / \mathrm{mL}$ for A2587T, and 444 $\pm 35 \mathrm{ng} / \mathrm{mL}$ for Natchez. These concentrations were much lower than the values obtained from fresh berries of the same genotypes by SPME analysis, 2,419 $\mathrm{ng} / \mathrm{mL}$ for A2528T, 5,574 $\mathrm{ng} / \mathrm{mL}$ for A2587T, and 3,882 $\mathrm{ng} / \mathrm{mL}$ for Natchez (data not shown). Qian and Wang (2005) reported total volatile concentrations of 3,950 and $24,540 \mathrm{ng} / \mathrm{mL}$ for Marion and Thornless Evergreen blackberries, respectively and total volatile concentrations of $12,885 \mathrm{ng} / \mathrm{mL}$ and 17,045 ng/mL were reported for Marion and Black Diamond blackberries, respectively ( $\mathrm{Du}$ et al. 2010). The lower volatile concentrations of extracts obtained by low temperature vacuum distillation compared with those of fresh blackberries are due to a dilution effect, i.e. addition of water to the berries to facilitate blending into a homogeneous puree and/or incomplete recovery of the volatiles at the low distillation temperure of $50^{\circ} \mathrm{C}$. Although the low temperature distillation method resulted in lower recovery of volatile compounds compared with fresh berry extracts measured by SPME, the technique helped prevent thermal degradation and iomerization of volatile compounds, especially monoterpenes. Thermal degradation of terpenes can occur via four different oxidative reactions, cleavage of double bonds, epoxidation, dehydrogenation into aromatic systems, and allylic oxidation into alcohols, ketones, and aldehydes (McGraw et al., 1999).

In the present study, the major class of volatiles were monoterpenes, alcohols, and esters, with volatile composition varing among the three genotypes. Monoterpenes accounted for $28 \%, 46 \%$ and $38 \%$ of total volatiles in A2528T, A2587T and Natchez, repectively, alcohols accounted for $26 \%, 34 \%$ and $31 \%$ of total volatiles in A2528T, A2587T and Natchez, repectively and esters accounted for $30 \%, 6 \%$, and $16 \%$ of total volatiles in A2528T, A2587T and
Natchez, repectively. Gu et al. (2020) reported acids and alcohols as the major classes in an unknown blackberry genotype, while Du et al. (2010) and Qian and Wang (2005) reported acids, alcohols, monoterpenes and furanones to be the major classes of volatiles in Marion, Black Diamond and Thornless Evergreen blackberries grown in Pacific Northwest. The differences in volatile composition between studies were not surprising since they can vary due to differences in genetics, maturation, sample preparation and GC conditions.

A total of 80 volatiles were identified among the three genotypes (Table 1). In A2528T, the five major volatiles were ethyl acetate $(76 \pm 11 \mathrm{ng} / \mathrm{mL})$, hexanol $(26 \pm 1 \mathrm{ng} / \mathrm{mL}), \alpha$-terpineol $(24$ $\pm 13 \mathrm{ng} / \mathrm{mL}), 2$-hexen-1-ol $(17 \pm 2 \mathrm{ng} / \mathrm{mL})$ and $\beta$-damascenone $(11 \pm 7 \mathrm{ng} / \mathrm{mL})$. In A2587T, they were 2-heptanol $(190 \pm 28 \mathrm{ng} /$ $\mathrm{mL}), \alpha$-terpineol $(69 \pm 14 \mathrm{ng} / \mathrm{mL})$, terpinen-4-ol $(53 \pm 4 \mathrm{ng} / \mathrm{mL})$, $\delta$-carvone $(52 \pm 8 \mathrm{ng} / \mathrm{mL})$ and ethyl acetate $(50 \pm 38 \mathrm{ng} / \mathrm{mL})$. In Natchez, they were ethyl acetate $(67 \pm 13 \mathrm{ng} / \mathrm{mL}), 6$-camphenol (40 $\pm 4 \mathrm{ng} / \mathrm{mL}), \alpha$-terpineol $(36 \pm 4 \mathrm{ng} / \mathrm{mL})$, terpinen-4-ol $(35 \pm 5$ $\mathrm{ng} / \mathrm{mL})$ and 1-hexanol $(35 \pm 5 \mathrm{ng} / \mathrm{mL})$.

\subsection{Antioxidant capacity of phenolic and volatile extracts}

The antioxidant capacities of the blackberry phenolic extracts were $30 \pm 1 \mu \mathrm{mol} / \mathrm{mL}$ TE for A2528T, $24 \pm 1 \mu \mathrm{mol} / \mathrm{mL}$ TE for A2587T and $25 \pm 2 \mu \mathrm{mol} / \mathrm{mL}$ TE for Natchez (Table 2). These values were higher than the previous value of $19.8 \mu \mathrm{mol} / \mathrm{mL}$ TE reported for an unknown blackberry variety (Gu et al., 2020). The volatile extracts had no antioxidant activity, which was expected since they are present in low concentration, and did not contain phenolics, especially anthocyanins that are highly correlated with antioxidant activities 


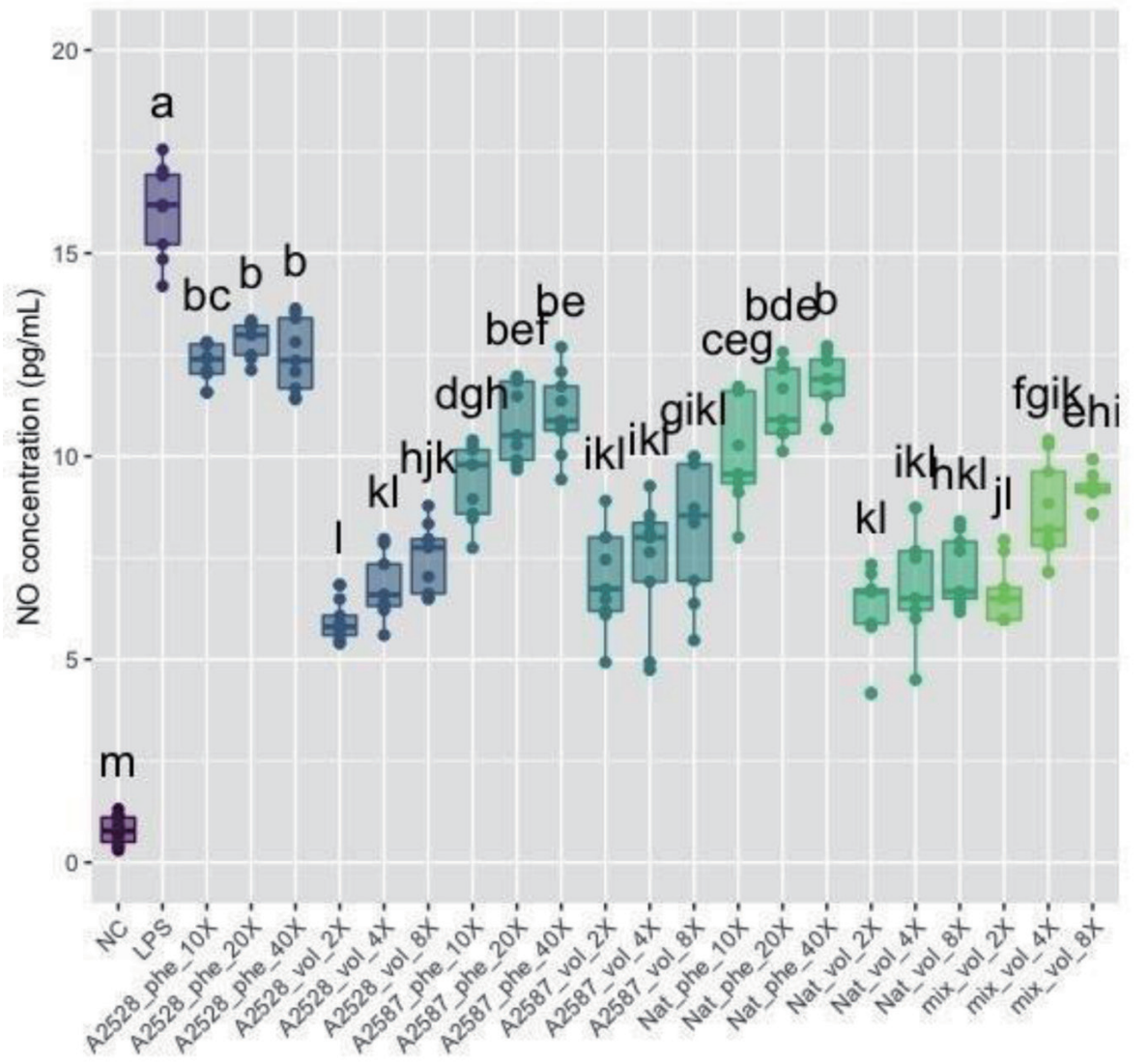

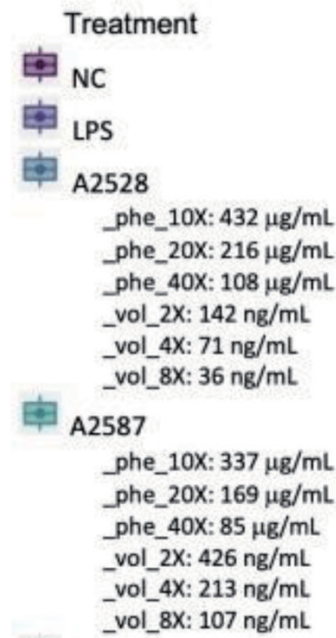

Nat

phe_10X: $368 \mu \mathrm{g} / \mathrm{mL}$ phe_20X: $184 \mu \mathrm{g} / \mathrm{mL}$ phe_40X: $92 \mu \mathrm{g} / \mathrm{mL}$ vol_2X: $222 \mathrm{ng} / \mathrm{mL}$ _vol_4X: $111 \mathrm{ng} / \mathrm{mL}$ _vol_8x: $56 \mathrm{ng} / \mathrm{mL}$

Mix

_vol_2X: $204 \mathrm{ng} / \mathrm{mL}$ vol_4X: $102 \mathrm{ng} / \mathrm{mL}$ _vol_8X: $51 \mathrm{ng} / \mathrm{mL}$

Figure 2. Average concentration of nitric oxide (NO, $\mu \mathrm{mol} / \mathrm{L}$ ) produced by RAW264.7 cells after a preventive treatment of one hr with phenolic extracts ("Phe") diluted 10-, 20-, or 40-fold or volatile extracts ("Vol") diluted 2-, 4-, or 8-fold, followed by LPS stimulation for 24 hr. The negative control ("NC") corresponds to cells without treatment nor LPS stimulation. The positive control ("LPS") corresponds to cells with LPS stimulation, but no treatment. The "Mix" is a lab-made solution of standard volatiles based on the average composition of the three blackberry volatile extracts. Conditions not connected by the same letter are significantly different $(\mathrm{P}<0.05)$.

of blackberry fruits (Kaume et al., 2012).

\subsection{Effect of blackberry extracts on cell viability}

The potential cytotoxicity of the phenolic and volatile extracts was measured with the MTS assay (Figure 1). None of the extracts significantly reduced cell viability compared with the non-treated cells, demonstrating that the extracts were not cytotoxic and that the effects observed in the following experiments were not due to a cytotoxic effect of blackberry phenolics or volatiles.

\subsection{Effect of blackberry extracts on NO production}

NO is an inflammatory mediator released by immune cells. Macrophages can release high levels of NO following pro-inflammatory cytokine or pathogen activation, whose roles include vasodilatation, modulation of the immune response and regulation of apoptosis (Sharma et al., 2007). The concentration in NO observed within the control cells and the cells treated with blackberry extracts are presented in Figure 2. The negative control corresponded to cells that were not treated and not inflamed with LPS, the positive control corresponded to cells that were not treated but inflamed. The average concentrations of NO in all dilutions of both phenolic and volatile extracts of all three genotypes were significantly lower than the positive control $(\mathrm{p}<0.05)$.

The percentage inhibition of NO was $24 \%, 42 \%$ and $37 \%$ by the phenolic extracts at 10 -fold dilution for A2528T, A2587T and Natchez, respectively, and $63 \%, 51 \%$ and $61 \%$ within the volatile extracts at the 2 -fold dilution, respectively. The average total phenolic concentrations in the 10 -fold diluted phenolic extracts were 432, 337 and $368 \mu \mathrm{g} / \mathrm{mL}$ for A2528T, A2587T and Natchez, respectively, while the average total volatile concentrations in the 2 -fold diluted volatile extracts were 142,426 , and $222 \mathrm{ng} / \mathrm{mL}$.

Treatments with blackberry phenolics effectively reduced NO synthesis in LPS-activated macrophages (Cuevas-Rodríguez et al., 2010; Pergola et al., 2006; Van de Velde et al., 2016, 2019). The potency of the NO lowering effect of blackberry phenolic extracts differed between genotypes, with better inhibition reported for genotypes containing higher phenolic concentrations (CuevasRodríguez et al., 2010; Van de Velde et al., 2016). Blackberry anthocyanins, and particularly cyanidin 3 -glucoside which is the dominant anthocyanin in blackberry, were particularly effective at reducing NO production of $\mathbf{J} 774$ murine monocyte/macrophages cells in a dose-dependent manner (Pergola et al., 2006). However, a later study comparing blackberry phenolic fractions demonstrated a better NO reduction effect of the proanthocyanidins $(25 \%$ inhibition) compared to the anthocyanin fraction (14\% inhibition) in RAW 264.7 cell induced with LPS (Van de Velde et al., 2019). 
Reduction of NO production by blackberry phenolic extracts is associated with the downregulation of the expression and/or activity of iNOS (Cuevas-Rodríguez et al., 2010; Pergola et al., 2006; Van de Velde et al., 2019), an enzyme responsible for the production of $\mathrm{NO}$ and upregulated during inflammation (Lowenstein and Padalko, 2004). iNOS synthesis itself can be initiated through the activation of the NF- $\kappa$ B pathway (Taylor and Geller, 2000), which has been shown to be partially inhibited by blackberry phenolic extracts (Gu et al., 2020; Pergola et al., 2006). Part of the NO reduction in the stimulated cells may also be attributed to the antioxidant activity of phenolic compounds and their capacity to scavenge reactive oxygen species (Basu and Maier, 2016).

Volatiles, in particular monoterpene compounds, have been studied for their anti-inflammatory effects although the literature mostly focused on tree terpenes and essential oil matrices (de Cássia da Silveira e Sá et al., 2013; Kim et al., 2020). Few studies have been conducted regarding food volatiles, but papers report the antiinflammatory effects of individual monoterpenes. In our blackberry volatile extracts, $\alpha$-terpineol was one of the major monoterpenes in all three genotypes. $\alpha$-terpineol has been used as treatment in LPS-induced murine macrophages, and was effective at reducing NO production at all concentrations tested $(1,10$ and $100 \mu \mathrm{g} /$ $\mathrm{ml}$ ), although no dose-response relationship was established (de Oliveira et al., 2012). $\alpha$-terpineol tested in RAW 264.7 cells at 1.16 $\mu \mathrm{g} / \mathrm{ml}$ also significantly reduced NO production by LPS-induced cells, although the volatile extract from cranberry was more effective than the monoterpenes tested alone (Moore et al., 2019). $\alpha$-terpineol has also been reported to inhibit NF- $\mathrm{BB}$ translocation in cancer cells (Hassan et al., 2010), along with other terpenoid compounds (Salminen et al., 2008). Similarly to the phenolic compounds, the ability of volatiles to reduce NO synthesis may be related to the capacity of few or several volatile compounds to inhibit the NF- $\mathrm{BB}$ pathway, and is supported by Gu et al. (2020) results who reported similar NO and NF-kB p65 inhibition between blackberry phenolic and volatile extracts. However, the decrease of NO synthesis by blackberry volatile extract through scavenging activity is not supported by our results, which showed no antioxidant effect of the blackberry volatile extracts.

Although our three blackberry genotypes varied in phenolic and volatile concentrations, all three showed comparable ability to inhibit secretion of NO induced by LPS. Our findings also suggest a much higher capacity for blackberry volatiles than phenolics to reduce NO synthesis, because they showed similar NO reduction activity, but at much lower concentration. This trend was previously observed in RAW 264.7 cells treated with phenolic and volatile fractions extracted from cranberries (Moore et al., 2019) and multiple berries including blackberries (Gu et al., 2020). This could suggest different mechanisms of action through which polyphenols and volatiles modulate pro-inflammatory pathways and ultimately the production of NO by macrophages, with a better efficacy of the volatiles. The lower molecular weight and reduced polarity of monoterpenes compared to phenolics make them better candidates to potentially interact with or enter the cells, where they could modulate different pathways involved in inflammation regulation.

The mixture of volatile standards simulating the major volatiles in the blackberry extract diluted 2-fold showed comparable ability to inhibit NO (59\%) as the volatile extracts from the three blackberry genotypes diluted 2-fold. Additionally, the mixture of volatile standards diluted 4 and 8 -fold resulted in $47 \%$ and $43 \%$ inhibition of NO production, respectively. This finding supports the hypothesis that the inhibitory effect of the blackberry volatile extracts on LPS-stimulated NO production was due to volatiles and not other compounds co-extracted with volatiles during the low temperature distillation protocol used to prepare the volatile extracts.

\subsection{Effects of blackberry extracts on IL-6 production}

IL-6 is a proinflammatory cytokine whose production can be induced in macrophages by LPS through the activation of the NF- $\mathrm{BB}$ pathway (Lee et al., 2015). The concentrations in IL-6 observed within the control cells and the cells treated with blackberry extracts are presented in Figure 3. The average concentrations in IL-6 in all dilutions of both phenolic and volatile extracts obtained from all three genotypes were significantly lower than the positive control $(p<0.05)$. The mix of standard volatiles which was made of volatile standards based on the volatile composition of the blackberry extracts showed the greatest ability to inhibit IL-6.

The percentage inhibition of IL- 6 within the phenolic extracts at the 10-fold dilution of A2528T, A2587T and Natchez was 50\%, $35 \%$ and $53 \%$, respectively, and $49 \%, 34 \%$ and $60 \%$ within the volatile extracts at the 2 -fold dilution, respectively. The inhibitory effect of both phenolic and volatile extracts was similar despite marked differences in bioactive compounds concentration. For example, the average bioactive compound concentration was of $337 \mu \mathrm{g} / \mathrm{mL}$ in phenolic extracts (10-fold dilution) of A2587T was 791 -fold higher than the $426 \mathrm{ng} / \mathrm{mL}$ in the respective volatile extract (2-fold dilution), yet both extracts showed comparable ability to prevent IL-6 production. The mix of standard volatiles showed remarkable ability to inhibit secretion of IL-6, with $81 \%, 77 \%$ and $72 \%$ inhibition at 2, 4 and 8 -fold dilutions, respectively.

As for NO, the inhibition of IL- 6 secretion by blackberry phenolics has been demonstrated in LPS-induced macrophages (Van de Velde et al., 2016). The comparison of IL-6 inhibition by three blackberry cultivars showed better inhibitory effect for the two genotypes higher in total polyphenolics, as already observed for the NO marker (Van de Velde et al., 2016). When investigating different blackberry phenolic fractions, however, the authors did not report a significant difference between blackberry crude extract, anthocyanin and proanthocyanidin fractions on IL-6, while the latter fraction was the most effective at reducing NO (Van de Velde et al., 2016). These observations suggest a similar potency of different blackberry phenolic fractions on the pro-inflammatory IL-6 marker, while the blackberry proanthocyanidins may have exerted better scavenging activity towards NO.

$\alpha$-terpineol, found in all three blackberry genotypes, and terpinen-4-ol, a major volatile in the A2587T and Natchez blackberries, are also two main components of tea tree essential oils (Nogueira et al., 2014) and have been studied for their anti-inflammatory effects on LPS-induced human macrophages. Terpinen-4-ol inhibited the production of IL-6 in macrophages induced with LPS from Escherichia coli at the two doses tested (551 and $68.1 \mu \mathrm{g} / \mathrm{ml})$, but $\alpha$-terpineol was only effective at the highest dose tested $(551 \mu \mathrm{g} /$ $\mathrm{ml}$ ) and had no effect at the lowest dose $(68.1 \mu \mathrm{g} / \mathrm{ml}$ ) (Nogueira et al., 2014). The effect of terpinen-4-ol was later tentatively explained through its modulatory effect towards the ERK mitogenactivated protein kinase (MAPK) pathway, while the whole tea tree extract slightly inhibited both NF- $\mathrm{kB}$ and MAPK (Nogueira et al., 2014). However, in KB cancer cell line, an epidermal carcinoma of the mouth, $\alpha$-terpineol effectively reduced the production of IL-6, as well as decreased the expression level of the IL-6 receptor gene in the cells. The other monoterpenes tested in this study, limonene and linalool, either increased (limonene) or did not impact IL-6 production (linalool) (Held et al., 2007). Our results support the modulation of IL-6 production by blackberry phytochemicals, and particularly volatile compounds. The inhibitory ef- 

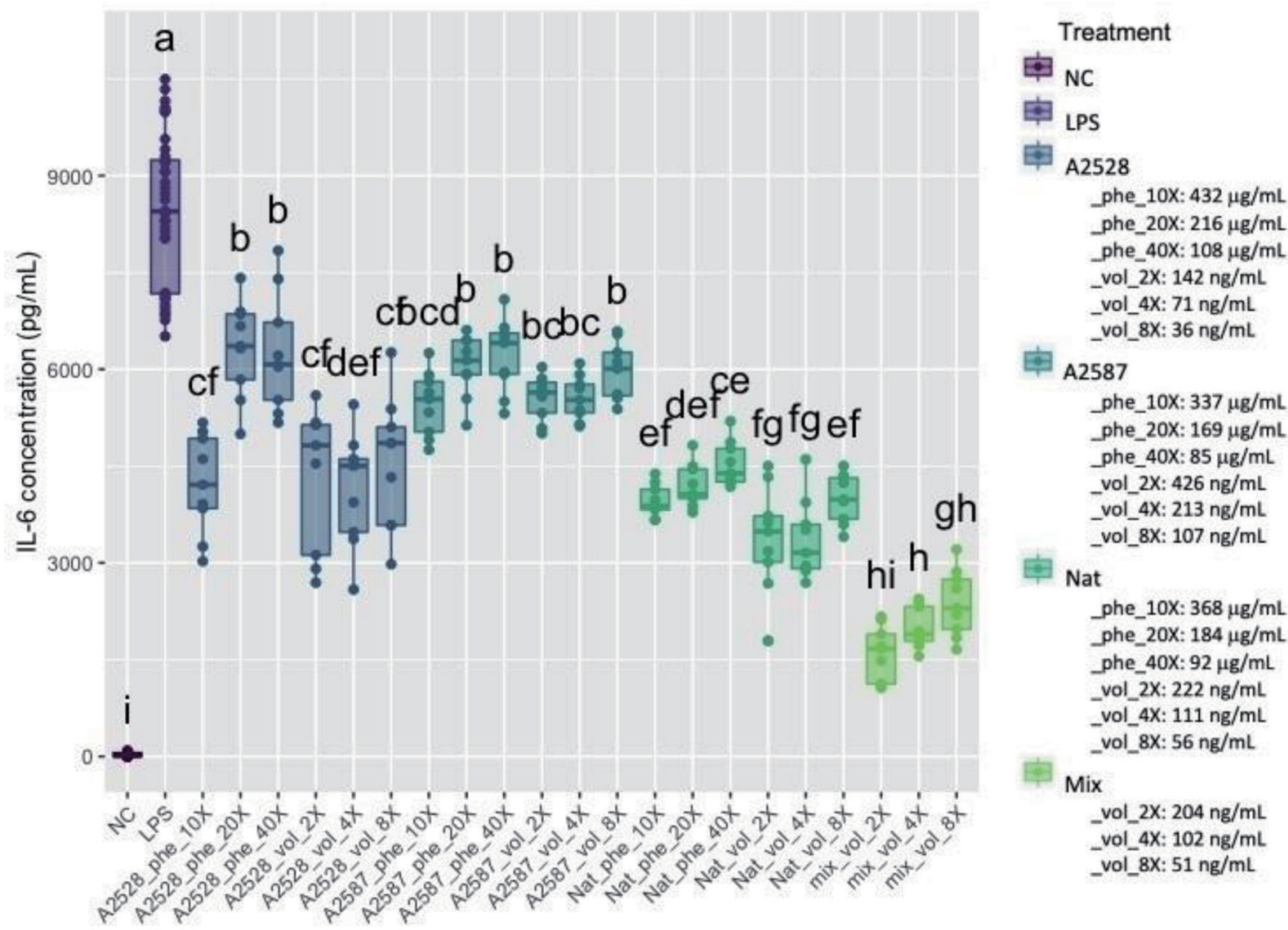

Figure 3. Average concentration of IL-6 (pg/ml) produced by RAW264.7 cells after a preventive treatment of one hr with phenolic extracts ("Phe") diluted 10-, 20-, or 40-fold or volatile extracts ("Vol”) diluted 2-, 4-, or 8-fold, followed by LPS stimulation for $\mathbf{2 4} \mathrm{hr}$. The negative control ("NC") corresponds to cells without treatment nor LPS stimulation. The positive control ("LPS") corresponds to cells with LPS stimulation, but no treatment. The "Mix" is a labmade solution of standard volatiles based on the average composition of the three blackberry volatile extracts. Conditions not connected by the same letter are significantly different $(\mathrm{P}<0.05)$.

fect of the volatiles (either extracted from blackberry or made from standards) towards IL-6 may be carried out by a few individual compounds with high potency towards IL-6 inhibition, and/or a synergistic effect between several compounds. More research is needed to identify the specific phenolic and volatile compounds that inhibit secretion of IL-6 and to determine potential synergistic effects among compounds, although the complexity of the volatile composition of blackberry complicates the task.

Similarly to what was previously observed within the NO assay, our results indicate a potentially much higher capacity for volatiles to prevent inflammation. A similar conclusion was made by $\mathrm{Gu}$ et al. (2020) after a blackberry volatile extract (35\% inhibition, average volatiles concentration of $244 \mathrm{ng} / \mathrm{mL}$ in cell media) exerted a higher IL-6 inhibitory effect than a blackberry phenolic extract ( $15 \%$ inhibition, average phenolics concentration of $77 \mathrm{ug} / \mathrm{mL}$ in cell media). Despite differences in the chemical profiles of the three blackberry genotypes tested in this study, all three genotypes showed comparable ability to inhibit secretion of IL-6 during the inflammatory process, strengthening the hypothesis of the compounds having synergistic effects rather than the extracts having only one main bioactive molecule.

\subsection{Effects of blackberry extracts on TNF- $\alpha$ production}

TNF- $\alpha$ is a cytokine that is rapidly produced after exposition to proinflammatory stimuli, and has various functions in coordinating the inflammatory response (Parameswaran and Patial, 2010), including the induction of iNOS expression in the liver in response to LPS (Salkowski et al., 1997). The NF-kB pathway is involved in the transcription of the TNF- $\alpha$ gene in LPS-induced macrophages (Shakhov et al., 1990).

The concentration in TNF- $\alpha$ observed within the control cells and the cells treated with blackberry extracts is presented in Figure 4 . The average concentration in TNF- $\alpha$ in all conditions except the 40-fold-diluted phenolic extracts was significantly lower than the positive control $(p<0.05)$. The percentage of inhibition of TNF- $\alpha$ secretion within the phenolic extracts at the 10 -fold dilution of A2528T, A2587T and Natchez was $31 \%, 44 \%$ and $28 \%$, respectively, and $61 \%, 73 \%$ and $67 \%$ within the volatile extracts at the 2 -fold dilution, respectively. The mix of standard volatiles again showed remarkable ability to inhibit secretion of TNF- $\alpha$, with $76 \%, 74 \%$ and $64 \%$, inhibition at 2,4 and 8 -fold dilutions, respectively, which confirmed that the observed results were due to the volatiles present in the extracts.

The effect of blackberry phenolic compounds on inflammation has mostly been measured using IL- 6 , IL-1 $\beta$, PGE $_{2}$ and NO markers, but few studies have reported the effect of blackberry phenolic fractions on TNF- $\alpha$ in macrophages in vitro. Gu et al. (2020) demonstrated a slight but significant reduction in TNF- $\alpha$ produced by LPS-induced RAW 264.7 cells following a treatment with blackberry phenolic fraction $(77 \mu \mathrm{g} / \mathrm{ml})$. Cyanidin 3-gluco- 


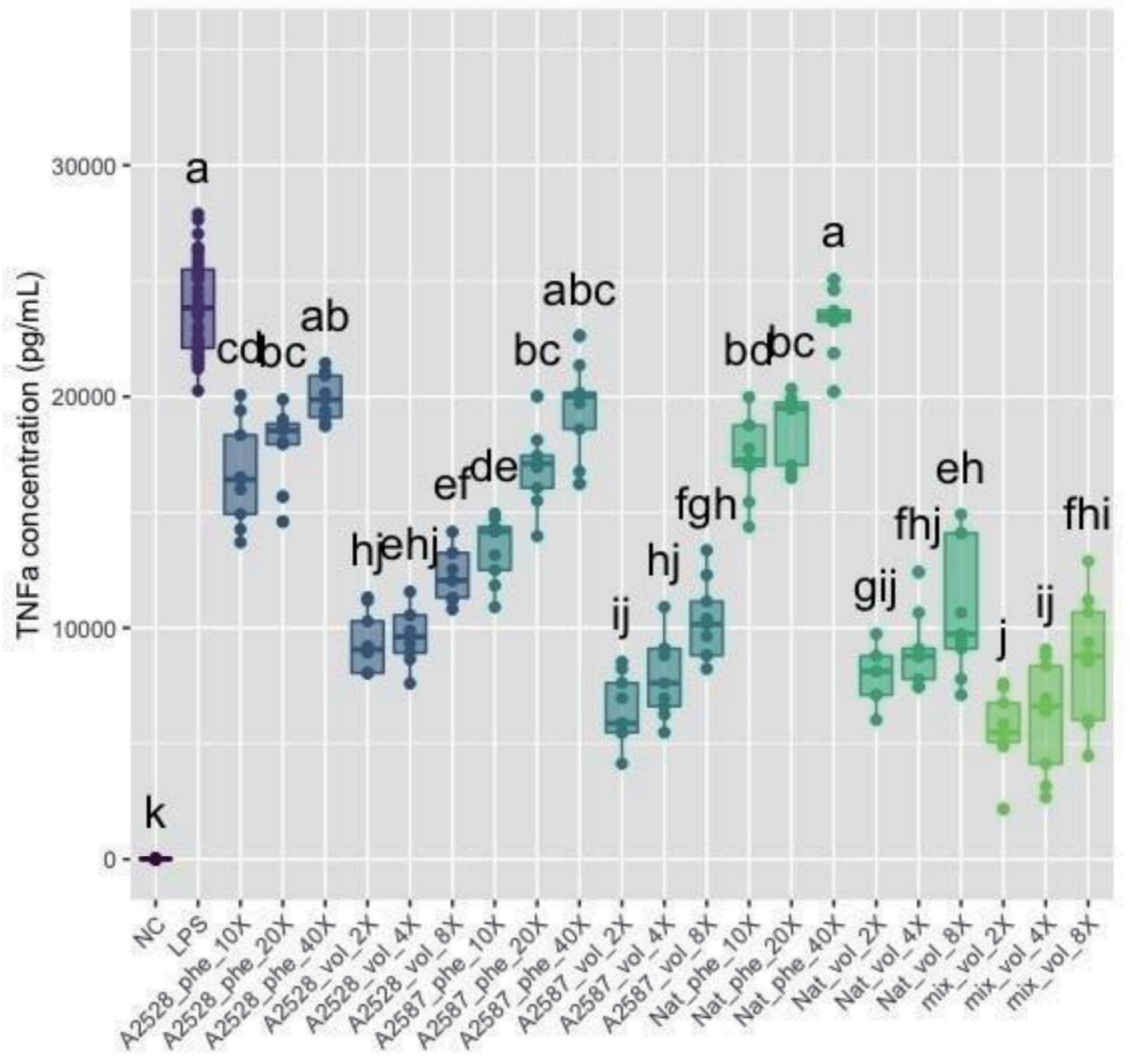

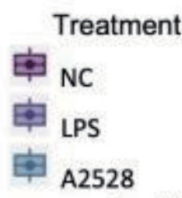

phe_10X: $432 \mu \mathrm{g} / \mathrm{mL}$ phe_20X: $216 \mu \mathrm{g} / \mathrm{mL}$ phe_40X: $108 \mu \mathrm{g} / \mathrm{mL}$ _vol_2X: $142 \mathrm{ng} / \mathrm{mL}$ vol_4X: $71 \mathrm{ng} / \mathrm{mL}$ _vol_8X: $36 \mathrm{ng} / \mathrm{mL}$

A2587 _phe_10X: $337 \mu \mathrm{g} / \mathrm{mL}$ phe_20X: $169 \mu \mathrm{g} / \mathrm{mL}$ phe_40X: $85 \mu \mathrm{g} / \mathrm{mL}$ _vol_2X: $426 \mathrm{ng} / \mathrm{mL}$ _vol_4X: $213 \mathrm{ng} / \mathrm{mL}$ _vol_8X: $107 \mathrm{ng} / \mathrm{mL}$

\section{Nat}

phe_10X: $368 \mu \mathrm{g} / \mathrm{mL}$ phe_20X: $184 \mu \mathrm{g} / \mathrm{mL}$ phe_40X: $92 \mu \mathrm{g} / \mathrm{mL}$ vol_ $2 x: 222 \mathrm{ng} / \mathrm{mL}$ _vol_4X: $111 \mathrm{ng} / \mathrm{mL}$ _vol_8X: $56 \mathrm{ng} / \mathrm{mL}$

Mix

_vol_2X: $204 \mathrm{ng} / \mathrm{mL}$ _vol_4X: $102 \mathrm{ng} / \mathrm{mL}$ _vol_ $8 x: 51 \mathrm{ng} / \mathrm{mL}$

Figure 4. Average concentration of TNF- $\alpha(\mathrm{pg} / \mathrm{ml})$ produced by RAW264.7 cells after a preventive treatment of one hr with phenolic extracts ("Phe”) diluted 10-, 20-, or 40-fold or volatile extracts ("Vol”) diluted 2-, 4-, or 8-fold, followed by LPS stimulation for 24 hr. The negative control ("NC") corresponds to cells without treatment nor LPS stimulation. The positive control ("LPS") corresponds to cells with LPS stimulation, but no treatment. The "Mix" is a lab-made solution of standard volatiles based on the average composition of the three blackberry volatile extracts. Conditions not connected by the same letter are significantly different $(\mathrm{P}<0.05)$.

side, the major anthocyanin reported in blackberry, significantly decreased TNF- $\alpha$ gene expression and protein synthesis in THP-1 human macrophages (Zhang et al., 2010). The inhibition effect was dose-dependent at concentrations ranging from 0.005 to $0.5 \mu \mathrm{M}$, but the effect was lost when the concentration was increased to 10 $\mu \mathrm{M}$ (Zhang et al., 2010).

LPS-induced human monocytes/macrophages treated with $\alpha$-terpineol did not show a reduction in TNF- $\alpha$ production (Hart et al., 2000; Nogueira et al., 2014). Terpinen-4-ol significantly decreased the production of TNF- $\alpha$ in human monocytes (Hart et al., 2000), but failed to regulate the same cytokine in U937 activated macrophages, which contradicted the observations reported in the same study on IL-6 (Nogueira et al., 2014). Although these two compounds show no or moderate activities towards TNF- $\alpha$, other monoterpenes were effective at reducing cytokine production in RAW 264.7 cells induced by LPS. Terpinolene and $\alpha$-phellandrene decreased TNF- $\alpha$ production by about 20 to $40 \%$ at 14 and $28 \mu \mathrm{g} /$ $\mathrm{ml}$, respectively (de Christo Scherer et al., 2019), and linalool used between 40 and $120 \mu \mathrm{g} / \mathrm{ml}$ dampened TNF- $\alpha$ production in a dosedependent manner, up to $70 \%$ inhibition (Huo et al., 2013).

Similarly to NO and IL- 6 results, the TNF- $\alpha$ inhibitory effect of our blackberry volatile extracts was stronger than phenolic extracts for all three genotypes, demonstrating a much higher capacity for volatiles to prevent inflammation. The conclusion was consistent with a previous study by Gu et al. (2020) where blackberry volatile extract showed higher ability to inhibit TNF- $\alpha$ than blackberry phenolic extract. Our results show a strong decrease in TNF- $\alpha$ production by RAW 264.7 cells treated with the blackberry volatile extract and volatile standard mix. Similarly to the previous tests, there was not enough evidence to claim a difference in effect between the three blackberry genotypes despite differences in phenolic and volatile composition among genotypes. The particular effect of one or several volatile compounds remain to be investigated, as the most bioactive compounds may not be the ones present at higher concentration in the extracts.

\section{Conclusion}

Blackberry phenolic and volatile fractions and a standard mix of blackberry volatile standards effectively inhibited the secretion of NO, IL-6 and TNF- $\alpha$ following LPS-induced inflammation of RAW264.7 cells. The volatile fraction showed greater ability to inhibit secretion of inflammatory cytokines than the phenolic fraction, despite being present at a much lower concentration. The three blackberry genotypes that varied in total volatile content all showed comparable ability to inhibit secretion of cytokines, indicating that volatile composition may be more important than total volatile content in modulating production of inflammatiory cytokines. Additional research is needed to determine the specific volatiles or class of volatiles responsible for anti-inflammatory activity, potential synergism existing between volatiles and phe- 
nolics, and the difference in respective mechanisms of action explaining the higher anti-inflammatory potency reported for volatiles. Future animal and human trials are also needed to determine bioavailability of volatile compounds, identification of metabolites and confirmation of anti-inflammatory effects in vivo.

C3GE: Cyanidin 3-glucoside equivalents; DPPH : 2,2 diphenyl1-picrylhydrazyl; ELISA: Enzyme linked immunosorbent assay; GAE: Gallic acid equivalents; GC: Gas chromatography; GC-FID: Gas chromatography - flame ionization detection; GC-MS: Gas chromatography - mass spectrometery; HPLC: High pressure liquid chromatography; HPLC-MS: High pressure liquid chromatography - mass spectrometry; IL-6: Interluekin 6; LPS: Lipopolysaccharide; MAPK: Mitogen-activated protein kinase; NC: Negative control; NED : N-1-napthylethylenediamine dihydrochloride ; NF-kB: Nuclear factor of kappa light polypeptide gene enhancer in beta cells; NO: Nitric oxide; Phe: Phenolic extract; RE: Rutin equivalents; SPE: Solid phase extraction; SPME: Solid phase microextraction; TNF $\alpha$ : Tumor necrosis factor alpha; Vol: Volatile extract

\section{Acknowledgments}

We thank Dr. John Clark, Horticulture Department, University of Arkansas for providing samples of blackberry genotypes for the study.

\section{Conflict of interest}

There are no conflicts of interest to declare.

\section{Supplementary material}

Table s1. Anthocyanin composition $(\mu \mathrm{g} / \mathrm{mL})$ of the phenolic fractions from the three selected blackberry genotypes.

Table s2. Flavonol composition $(\mu \mathrm{g} / \mathrm{mL})$ of the phenolic fractions from the three selected blackberry genotypes.

\section{References}

Basu, P., and Maier, C. (2016). In vitro antioxidant activities and polyphenol contents of seven commercially available fruits. Pharmacog. Res. 8: 258-264.

Bowen-Forbes, C.S., Zhang, Y., and Nair, M.G. (2010). Anthocyanin content, antioxidant, anti-inflammatory and anticancer properties of blackberry and raspberry fruits. J. Food Comp. Anal. 23: 554-560.

Cho, M.J., Howard, L.R., Prior, R.L., and Clark, J.R. (2005). Flavonol glycosides and antioxidant capacity of various blackberry and blueberry genotypes determined by high-performance liquid chromatography/ mass spectrometry. J. Sci. Food Agric. 85: 2149-2158.

Cho, M.J., Howard, L.R., Prior, R.L., and Clark, J.R. (2004). Flavonoid glycosides and antioxidant capacity of various blackberry, blueberry and red grape genotypes determined by high-performance liquid chromatography/mass spectrometry. J. Sci. Food Agric. 84: 1771-1782.

Cuevas-Rodríguez, E.O., Dia, V.P., Yousef, G.G., García-Saucedo, P.A., LópezMedina, L., Paredes-López, O., Gonzalez de Mejia, E., and Lila, M.A. (2010). Inhibition of pro-inflammatory responses and antioxidant capacity of Mexican blackberry (Rubus spp.) extracts. J. Agric. Food Chem. 58: 9542-9548.

de Cássia da Silveira e Sá, R., Andrade, L.N., and de Sousa, D.P. (2013). A review on anti-inflammatory activity of monoterpenes. Molecules
18: $1227-1254$.

de Christo Scherer, M.M., Marques, F.M., Figueira, M.M., Peisino, M. Schmitt, E.F.P., Kondratyuk, T.P., Endringer, D.C., Scherer, R., and Fronza, M. (2019). Wound healing activity of terpinolene and $\alpha$-phellandrene by attenuating inflammation and oxidative stress in vitro. J. Tissue Viab. 28: 94-99.

de Oliveira, M.G.B., Marques, R.B., de Santana, M.F., Santos, A.B.D., Brito, F.A., Barreto, O., De Sousa, D.P., Almeida, F.R.C., Badauê-Passos, D. Antoniolli, J.A.R., and Quintans-Júnior, L.J. (2012). $\alpha$-terpineol reduces mechanical hypernociception and inflammatory response. Basic Clin. Pharmacol. Toxicol. 11: 120-125.

Du, X., Finn, C.E., and Qian, M.C. (2010). Volatile composition and odouractivity value of thornless 'Black Diamond' and 'Marion' Blackberries. Food Chem. 119: 1127-1134.

Gorinstein, S., Haruenkit, R., Park, Y-S., Jung, S-T., Zachwieja, Z., Jastrzebski, Z., Katrich, E., Trakhtenberg, S., and Belloso, O.M. (2004). Bioactive compounds and antioxidant potential in fresh and dried Jaffa ${ }^{\circledR}$ sweeties, a new kind of citrus fruit. J. Sci. Food Agric. 84: 1459-1463.

Gu, I., Brownmiller, C., Stebbins, N.B., Mauromoustakos, A., Howard, L., and Lee, S.-O. (2020). Berry phenolic and volatile extracts inhibit proinflammatory cytokine secretion in LPS-stimulated RAW264.7 cells through suppression of NF-KB signaling pathway. Antioxidants 9: 871.

Hart, P.H., Brand, C., Carson, C.F., Riley, T.V., Prager, R.H., and Finlay-Jones, J.J. (2000). Terpinen-4-ol, the main component of the essential oil of Melaleuca alternifolia (tea tree oil) suppresses inflammatory mediator production by activated human monocytes. Inflam. Res. 49: 619-626.

Hassan, S.B., Gali-Muhtasib, H., Göransson, H., and Larsson, R. (2010). Alpha terpineol: A potential anticancer agent which acts through suppressing NF-kappaB signalling. Anticancer Res. 30: 1911-1919.

Held, S., Schieberle, P., and Somoza, V. (2007). Characterization of alphaterpineol as an anti-inflammatory component of orange juice by in vitro studies using oral buccal cells. J. Agric. Food Chem. 55: 80408046

Hirota, R., Roger, N.N., Nakamura, H., Song, H.S., Sawamura, M., and Suganuma, N. (2010). Anti-inflammatory effects of limonene from yuzu (Citrus junos Tanaka) essential oil on eosinophils. J. Food Sci. 75: $\mathrm{H} 87-\mathrm{H} 92$.

Huo, M., Cui, X., Xue, J., Chi, G., Gao, R., Deng, X., Guan, S., Wei, J., Soromou, L., Feng, H., and Wang, D. (2013). Anti-inflammatory effects of linalool in RAW 264.7 macrophages and lipopolysaccharide-induced lung injury model. J. Surg. Res. 180: e47-e54.

Kaume, L., Howard, L.R., and Devareddy, L. (2012). The blackberry fruit: A review on its composition and chemistry, metabolism and bioavailability, and health benefits. J. Agric. Food Chem. 60: 5716-5727.

Kim, T., Song, B., Cho, K.S., and Lee, I.S. (2020). Therapeutic potential of volatile terpenes and terpenoids from forests for inflammatory diseases. Int. J. Molec. Sci. 21(6): 2187.

Kolniak-Ostek, J., Kucharska, A.K., Sokół-Łętowska, A., and Fecka, I. (2015). Characterization of phenolic compounds of thorny and thornless blackberries. J. Agric. Food Chem. 63: 3012-3021.

Lee, A.J., Cho, K.J., and Kim, J.H. (2015). MyD88-BLT2-dependent cascade contributes to LPS-induced interleukin- 6 production in mouse macrophage. Exp. Molec. Med. 47: e156.

Lowenstein, C.J., and Padalko, E. (2004). INOS (NOS2) at a glance. J. Cell Sci. 117: 2865-2867.

McGraw, G.W., Hemingway, R.W., Ingram, L.L., Canady, Jr, R.S., and McGraw, W.B. (1999). Thermal degradation of terpenes: camphene, $\Delta^{3}$-carene, limonene, and $\alpha$-terpinolene. Environ. Sci. Technol. 33 : 4029-4033.

Mikulic-Petkovsek, M., Slatnar, A., Stampar, F., and Veberic, R. (2012). HPLC-MSn identification and quantification of flavonol glycosides in 28 wild and cultivated berry species. Food Chem. 135: 2138-2146.

Moore, K., Howard, L., Brownmiller, C., Gu, I., Lee, S.-O., and Mauromoustakos, A. (2019). Inhibitory effects of cranberry polyphenol and volatile extracts on nitric oxide production in LPS activated RAW 264.7 macrophages. Food Func. 10: 7091-7102.

Nogueira, M.N.M., Aquino, S.G., Rossa Junior, C., and Spolidorio, D.M.P. (2014). Terpinen-4-ol and alpha-terpineol (tea tree oil components) inhibit the production of IL-1 $\beta$, IL- 6 and IL-10 on human macrophages. Inflammation Research 63: 769-778. 
Parameswaran, N., and Patial, S. (2010). Tumor necrosis factor- $\alpha$-signalling in macrophages. Crit. Rev. Eukaryot. Gene Exp. 20: 87-103.

Pergola, C., Rossi, A., Dugo, P., Cuzzocrea, S., and Sautebin, L. (2006). Inhibition of nitric oxide biosynthesis by anthocyanin fraction of blackberry extract. Nitric Oxide 15: 30-39.

Perkins-Veazie, P., Collins, J.K., and Clark, J.R. (1996). Cultivar and maturity affect postharvest quality of fruit from erect blackberries. HortScience 31: 258-261.

Plastina, P., Apriantini, A., Meijerink, J., Witkamp, R., Gabriele, B., and Fazio, A. (2018). In vitro anti-inflammatory and radical scavenging properties of Chinotto (Citrus myrtifolia Raf.) essential oils. Nutrients 10: 783.

Qian, M.C., and Wang, Y. (2005). Seasonal variation of volatile composition and odor activity value of 'Marion' (Rubus Spp. Hyb) and 'Thornless Evergreen' (R. Laciniatus L.) blackberries. J. Food Sci. 70: C13-C20.

Salkowski, C.A., Detore, G., McNally, R., van Rooijen, N., and Vogel, S.N. (1997). Regulation of inducible nitric oxide synthase messenger RNA expression and nitric oxide production by lipopolysaccharide in vivo: the roles of macrophages, endogenous IFN-gamma, and TNF receptor-1-mediated signaling. Immunology 158: 905-912.

Salminen, A., Lehtonen, M., Suuronen, T., Kaarniranta, K., and Huuskonen, J. (2008). Terpenoids: Natural inhibitors of NF-kappaB signaling with anti-inflammatory and anticancer potential. Cell. Molec. Life Sci. 65 2979-2999.

Seeram, N.O., Adams, L.S., Zhang, Y., Lee, R., Sand, D., Scheuller, H.S., and Heber, D. (2006). Blackberry, black raspberry, blueberry, cranberry, red raspberry, and strawberry extracts inhibit growth and stimulate apoptosis of human cancer cells in vitro. J. Agric. Food Chem. 54: 9329-9339.
Shakhov, A.N., Collart, M.A., Vassalli, P., Nedospasov, S.A., and Jongeneel, C.V. (1990). Kappa B-type enhancers are involved in lipopolysaccharide-mediated transcriptional activation of the tumor necrosis factor alpha gene in primary macrophages. J. Exp. Medic. 171: 5-47.

Sharma, J.N., Al-Omran, A., and Parvathy, S.S. (2007). Role of nitric oxide in inflammatory diseases. Inflammopharmacology 15: 252-259.

Slinkard, K., and Singleton, V.L. (1977). Total phenol analysis: automation and comparison with manual methods. Am. J. Enol. Viticul. 28: 49-55.

Strick, B. (2007). Berry crops: Worldwide area and production systems. In: Zhao, Y. (Ed.). Berry Fruit: Value-Added Products for Health Promotion. CRC Press, Boca Raton, pp. 3-49.

Taylor, B.S., and Geller, D.A. (2000). Molecular regulation of the human inducible nitric oxide synthase (iNOS) gene. Shock 13: 413-424.

Van de Velde, F., Esposito, D., Grace, M.H., Pirovani, M.E., and Lila, M.A. (2019). Anti-inflammatory and wound healing properties of polyphenolic extracts from strawberry and blackberry fruits. Food Res.Int. 121: 453-462.

Van de Velde, F., Grace, M.H., Esposito, D., Pirovani, M.T., and Lila, M.A. (2016). Quantitative comparison of phytochemical profile, antioxidant, and anti-inflammatory properties of blackberry fruits adapted to Argentina. J. Food Comp. Anal. 47: 82-91.

Wu, X., and Prior, R.L. (2005). Systematic identification and characterization of anthocyanins by HPLC-ESI-MS/MS in common foods in the United States: Fruits and berries. J. Agric. Food Chem. 53: 2589-2599.

Zhang, Y., Lian, F., Zhu, Y., Xia, M., Wang, Q., Ling, W., and Wang, X.D. (2010). Cyanidin-3-O-beta-glucoside inhibits LPS-induced expression of inflammatory mediators through decreasing IkappaBalpha phosphorylation in THP-1 cells. Inflam. Res. 59: 723-730. 